\title{
Plasma lipidome is dysregulated in Alzheimer's disease and is associated with disease risk genes
}

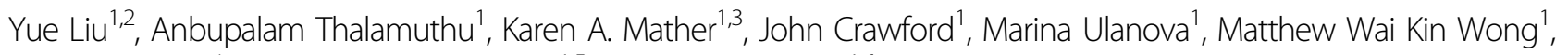
Russell Pickford ${ }^{4}$, Perminder S. Sachdev $\mathbb{D}^{1,5}$ and Nady Braidy $\mathbb{B D}^{1,6}$

\begin{abstract}
Lipidomics research could provide insights of pathobiological mechanisms in Alzheimer's disease. This study explores a battery of plasma lipids that can differentiate Alzheimer's disease (AD) patients from healthy controls and determines whether lipid profiles correlate with genetic risk for AD. AD plasma samples were collected from the Sydney Memory and Ageing Study (MAS) Sydney, Australia (aged range 75-97 years; 51.2\% male). Untargeted lipidomics analysis was performed by liquid chromatography coupled-mass spectrometry (LC-MS/MS). We found that several lipid species from nine lipid classes, particularly sphingomyelins (SMs), cholesterol esters (ChEs), phosphatidylcholines (PCs), phosphatidylethanolamines (PIs), phosphatidylinositols (PIs), and triglycerides (TGS) are dysregulated in AD patients and may help discriminate them from healthy controls. However, when the lipid species were grouped together into lipid subgroups, only the DG group was significantly higher in AD. ChEs, SMs, and TGs resulted in good classification accuracy using the Glmnet algorithm (elastic net penalization for the generalized linear model $[\mathrm{glm}]$ ) with more than 80\% AUC. In general, group lipids and the lipid subclasses LPC and PE had less classification accuracy compared to the other subclasses. We also found significant increases in SMs, Pls, and the LPE/PE ratio in human U251 astroglioma cell lines exposed to pathophysiological concentrations of oligomeric $A \beta_{42}$. This suggests that oligomeric $A \beta_{42}$ plays a contributory, if not causal role, in mediating changes in lipid profiles in AD that can be detected in the periphery. In addition, we evaluated the association of plasma lipid profiles with AD-related single nucleotide polymorphisms (SNPs) and polygenic risk scores (PRS) of AD. We found that FERMT2 and MS4A6A showed a significantly differential association with lipids in all lipid classes across disease and control groups. ABCA7 had a differential association with more than half of the DG lipids (52.63\%) and PI lipids (57.14\%), respectively. Additionally, 43.4\% of lipids in the SM class were differentially associated with CLU. More than $30 \%$ of lipids in ChE, PE, and TG classes had differential associations with separate genes (ChE-PICALM, SLC24A4, and SORL1; PE-CLU and CR1; TG-BINI) between AD and control group. These data may provide renewed insights into the pathobiology of $A D$ and the feasibility of identifying individuals with greater $A D$ risk.
\end{abstract}

\section{Introduction}

Alzheimer's disease (AD) is the most common cause of dementia, accounting for about $70 \%$ of total cases $^{1}$. This progressively neurodegenerative disease is characterized

\footnotetext{
Correspondence: Nady Braidy (n.braidy@unsw.edu.au)

${ }^{1}$ Centre for Healthy Brain Ageing (CHeBA), School of Psychiatry, University of New South Wales, Sydney, Australia

${ }^{2}$ Guangdong Mental Health Center, Guangdong Provincial People's Hospital, Guangdong Academy of Medical Sciences, Guangzhou, Guangdong, China Full list of author information is available at the end of the article
}

by an insidious onset and is clinically defined by a progressive loss of memory and other cognitive deficits. AD has become one of the major challenges for the public health and economic system of the 21st century. There is still no international consensus on the etiology of this multifactorial disease, in which in addition to proteinopathies, oxidative stress, inflammation, metabolic disorder, and other factors play a part ${ }^{2-4}$.

The lack of effective treatments and the potential for prevention highlight the importance of identifying early 
biomarkers for diagnosing AD. In addition, there is evidence that pathological processes associated with $\mathrm{AD}$ can also manifest in the peripheral system ${ }^{5}$, indicating the possibility of identifying non-invasive blood biomarkers. Lipids participate in important functions such as cell membrane formation, cellular transport, and energy storage, and act as essential signaling molecules. Beyond their structural roles, lipids have also been shown to act as modulators of transmembrane proteins, such as ion channels, whereby alteration of the composition or conformation of lipids surrounding ion channels can affect their function ${ }^{6,7}$. Given the essential role of lipids in major biological processes, blood lipids have emerged as promising biomarkers for $\mathrm{AD}^{8-10}$.

Although there have been many studies on the association between lipids and the pathobiology of $\mathrm{AD}$, there are few studies on the plasma lipidome in $\mathrm{AD}$. In contrast to classical biochemical approaches that focus on single metabolites or reactions, lipidomics approaches simultaneously identify and quantify hundreds of lipids. Measurement of large numbers of lipids enables network analysis approaches and provides means to identify critical metabolic drivers in disease pathophysiology. Lipidomics provides powerful tools for mapping global biochemical changes in disease and treatment.

In our current study, we examined differences in the plasma lipidome between $\mathrm{AD}$ and 'healthy' age-matched controls and compared the ability of different lipid profiles to discriminate between the two groups. We also showed that human astroglioma cultures exposed to pathological levels of amyloid-beta $(\mathrm{A} \beta)_{42}$ oligomers shared similar cellular lipidomic profiles to those observed in human plasma $\mathrm{AD}$. We explored the effect of $\mathrm{AD}$ polygenic risk scores and AD-related SNPs on plasma lipid levels between $\mathrm{AD}$ and controls.

\section{Method \\ Participants}

Participants were a subsample from the populationbased longitudinal Sydney Memory and Ageing Study $(\text { Sydney MAS })^{11}$, an ongoing study that began in 2005 and focuses on cognitive decline in community-dwelling elderly. Participants were aged 70-90 years, initially without dementia, living in the community, and able to complete their assessments in English. There have been four Waves of data collection, two years apart. At each Wave, participants underwent an MRI scan, comprehensive neuropsychological assessment, medical examination, and blood collection for biochemistry analyses and DNA extraction. Written informed consent was obtained from all participants. In this study, AD (diagnosed by NINCDSADRDA criteria) and cognitively normal control samples were collected at Waves 2 and 4. Ethics approval for this study was obtained from UNSW Sydney Australia and the South-Eastern Illawarra Area Health Service-Eastern sector $^{11}$. The investigators were blinded to sample allocation during the study and outcome assessment.

\section{Plasma lipid extraction}

Plasma lipids were extracted as previously described ${ }^{12}$. Briefly, $10 \mu \mathrm{L}$ internal lipid standards (ISTDs) (Avanti lipids, https://avantilipids.com/product/330707) were added to $10 \mu \mathrm{L}$ aliquot of each plasma sample. $100 \mu \mathrm{L}$ of 1-butanol-Methanol $(1: 1 \mathrm{v} / \mathrm{v})$ containing $5 \mathrm{mM}$ ammonium formate were used to dissolve the mixture. Samples were vortexed for $10 \mathrm{~s}$ then sonicated for one hour. Afterward, samples were centrifuged at $13,000 \times g$ for $10 \mathrm{~min}$. The supernatant was transferred into a fresh Eppendorf tube. A further $100 \mu \mathrm{L}$ of 1-butanol/methanol $(1: 1 \mathrm{v} / \mathrm{v})$ with $5 \mathrm{mM}$ ammonium formate was added to the white pellet to re-extract any remaining lipids. The supernatant was dried in a speed vacuum centrifuge for 40-60 min. The lipids were reconstituted by adding $100 \mu \mathrm{L}$ of 1 -butanol/methanol $(1: 1 \mathrm{v} / \mathrm{v})$ containing $5 \mathrm{mM}$ ammonium formate to each tube. The contents were transferred into a $300 \mu \mathrm{L}$ glass Chromacol vial with a glass insert prior to liquid chromatography/mass spectrometry (LC-MS).

\section{Liquid chromatography/mass spectrometry}

Lipid analysis was performed by LC ESI-MS/MS using a Thermo QExactive Plus Orbitrap mass spectrometer as previously described ${ }^{12}$. Briefly, a Waters ACQUITY UPLC $\mathrm{CSH}^{\mathrm{TM}} \mathrm{C} 181.7 \mu \mathrm{m}, 2.1 \times 100 \mathrm{~mm}$ column was used for liquid chromatography at a flow rate of $260 \mathrm{gl} / \mathrm{min}$, using the following gradient condition: $32 \%$ solvent B to $100 \%$ over $25 \mathrm{~min}$, a return to $32 \% \mathrm{~B}$ and finally $32 \%$ B for $5 \mathrm{~min}$ prior to the next injection. Solvents A and B consisted of acetonitrile: MilliQ water $(6: 4 \mathrm{v} / \mathrm{v})$ and isopropanolacetonitrile (9:I v/v) respectively, both containing $10 \mathrm{mM}$ ammonium formate and $0.1 \%$ formic acid. The first $3 \mathrm{~min}$ of eluent, containing the eluted salts, was diverted to waste. A product ion scan in positive and negative ion modes was performed to analyze the individual lipid species. The order of sampling was randomized prior to analysis. Ceramide (Cer), sphingomyelin (SM), phosphatidylcholines (PC), phosphatidylethanolamines (PE), phosphatidylinositol (PI), lyso-phosphatidylcholines (LPC), cholesterol esters (ChE), diacylglycerol (DG) and triacylglycerols (TG) were detected. The abundance of lipids was acquired using Lipidsearch software version 4.2 (Thermo Fisher Scientific, Sydney, NSW AU) according to accurate lipid mass and fragment matching ${ }^{12}$. The LC-MS data were exported into Microsoft Excel and normalized by dividing the abundance 
of internal standards to be used for further statistical analyses.

\section{Cell lipidomics \\ Cell culture}

U251 human astroglioma cell lines were purchased from the ATCC. These cells were cultivated in Roswell Park Memorial Institute (RPMI) 1640 Medium supplemented with $10 \%$ fetal bovine serum, 1\% 1-glutamax, and $1 \%$ antibacterial/antifungal. The cells were recently tested and found to be mycoplasma-free using the MycoAlert Mycoplasma Detection Kit (Lonza). The cells were grown at $37{ }^{\circ} \mathrm{C}$ in $95 \%$ humidified air and $5 \% \mathrm{CO}_{2}$. The culture medium was replaced every 2 days. U251 cells were seeded in a 12-well microtitre plate and given treatment when cells were nearly at confluency $(0.5 \times 106$ cells per well). Groups for evaluation are as follows: 6 wells containing naive U251 cells with no treatment (control) for $24 \mathrm{~h}$, and 6 wells containing U251 cells treated for $24 \mathrm{~h}$ with $5 \mu \mathrm{M}$ oligomeric $\mathrm{A} \beta_{42}$ (see below for further details).

\section{Preparation of recombinant $A \beta_{42}$ peptide}

$\mathrm{A} \beta_{42}$ peptide was purchased from Recombinant Peptide Technologies (Athens, GA, USA). The peptide was immediately stored in sealed glass vials at $-80^{\circ} \mathrm{C}$ in a lyophilized form. Consistent homogenous preparations of recombinant $A \beta_{42}$ oligomers or fibrils for use in cell culture stimulation experiments were obtained following a previously published protocol ${ }^{13}$. To avoid condensation upon opening, each vial was left at room temperature for $30 \mathrm{~min}$ prior to resuspension. Using 1,1,1,3,3,3-hexafluoro-2-propanol (HFIP; Sigma, Castle Hill, Australia), the lyophilized peptide was initially dissolved to $1 \mathrm{mM}$ and separated into $50 \mu \mathrm{l}$ aliquots in sterile microcentrifuge tubes. Aliquots were left for $3 \mathrm{~h}$ in a fume hood, which allowed almost complete evaporation of HFIP. The resulting peptide films were further dried under vacuum using a Speed Vac (ThermoSavant, Patterson, CA, USA). This procedure is important as HFIP evaporated $A \beta$ will form fibrils if exposed to moisture in the air, hence it is paramount to store HFIP evaporated $A \beta$ films in desiccant. These preparations were then stored at $-20^{\circ} \mathrm{C}$ until required.

\section{Production of recombinant $A \beta_{42}$ oligomers}

Each HFIP aliquot of the peptide film was thoroughly resuspended to $5 \mathrm{mM}$ in anhydrous dimethyl sulfoxide (DMSO; Sigma, Castle Hill, Australia) via pipette mixing, followed by 10-min bath sonication (Model: FX8, Unisonics, Sydney, Australia). Oligomers were formed by adding ice-cold Dulbecco's modified Eagle medium/Ham F-12 without phenol red (Sigma, Castle Hill, Australia) to a final concentration of $100 \mu \mathrm{M}$. Following a $30 \mathrm{~s}$ vortex, the preparation was incubated at $4{ }^{\circ} \mathrm{C}$ for 6 weeks for the formation of $A \beta_{42}$ oligomers.

\section{Cell lipidomics}

Samples were collected using a cell scraper to $1 \mathrm{~mL}$ PBS solvent. After centrifuging $5 \mathrm{~min}$ at $13,000 \times g$, cell pellets were collected and reconstituted in $110 \mu \mathrm{L}$ MilliQ water. $10 \mu \mathrm{L}$ of cell solution was used for protein assay and the rest was used for lipid extraction. Lipids extraction and LC-MS methods were the same as plasma lipids, which were described above. 10 lipid classes including Cer, SM, PC, PE, PI, LPC, lyso-phosphatidylethanolamines (LPE), ChE, DG, and TG were detected.

\section{Quantification of oligomeric $A \beta$}

Oligomeric $A \beta$ was quantified as previously described ${ }^{14}$. Briefly, plasma samples were thawed at $37^{\circ} \mathrm{C}$ for $15 \mathrm{~min}$. Afterwards, $10 \mu \mathrm{l}$ of plasma, $4 \mu \mathrm{l}$ of HAMA (human antimurine antibody, HAMA) blocker (Scantibodies Laboratory, Santee, CA, USA), were mixed. $10 \mu \mathrm{l}$ of PBR-1 (1\% proprietary $+1.25 \%$ dimethyl sulfoxide (DMSO) + 96.75\% phosphate-buffered saline contains Tween 20 $(\mathrm{PBST})+1 \%$ ultra-pure water) were further mixed into the plasma mixture. The mixtures were incubated for 48 and $1 \mathrm{~h}$, respectively. The plasma sample mixture and serially diluted standards were added to separate wells of the plate in a total volume of $100 \mu \mathrm{l}$. The plates were incubated at RT for $1 \mathrm{~h}$. The detection antibody was added to the wells, and the plate was incubated for $1 \mathrm{~h}$ at RT. Finally, $100 \mu \mathrm{l}$ of $3,3^{\prime}, 5,5^{\prime}$-tetramethylbenzidine (TMB) reagent was added as a substrate, and after $15 \mathrm{~min}$, the reaction was stopped with $50 \mu \mathrm{l}$ of $1 \mathrm{M} \mathrm{H}_{2} \mathrm{SO}_{4}$. Optical density (OD) values were measured using the BMG Fluostar Optima multimode plate reader (NY, USA), at a wavelength of $450 \mathrm{~nm}$. Prior studies using this method detected the raw luminescence signal and used relative luminescence units (RLU) to present the oligomeric $A \beta$ levels ${ }^{15}$.

\section{Genotyping}

DNA was extracted using standard procedures. Genotyping of the $A P O E \varepsilon 2 / 3 / 4$ polymorphism was performed as described ${ }^{16}$. Genome-wide genotyping was undertaken using the Affymetrix Genome-wide Human SNP Array 6.0 (California, USA) at the Ramaciotti Centre, UNSW Australia ${ }^{17}$. The CRLMM package (v1.10.0) in R (v2.12.1) was used to call genotypes. SNPs were excluded if the genotyping call rate was $<95 \%$, had a minor allele frequency $<0.01$ or if they failed a Hardy-Weinberg equilibrium threshold of $<1 \times 10^{-6}$. After further QC checks, there were 925 Sydney MAS participants with data for 734,550 SNPs. Imputation was undertaken to the 1000 Genome reference panel using the Michigan Imputation 
Server. SNPs with poor imputation quality were omitted from any further analyses $\left(R^{2} \leq 0.6\right)$.

\section{Polygenic Risk Scores (PRS) and AD implicated SNPs}

PRS were generated using the PRSice program ${ }^{18}$ from summary statistics obtained from a previous Alzheimer's disease GWAS $^{19}$. Linkage disequilibrium pruning was performed using the clumping option $\left(r^{2}>0.25\right.$ and physical distance threshold of $250 \mathrm{~kb} \mathrm{~KB}$ ). We present the association of lipids with the PRS calculated using the SNPs with the AD GWAS $p$-value threshold $\leq 5 \times 10^{-5}$. Associations between 33 individual AD-related $\mathrm{SNPs}^{20}$ that passed QC checks (SNPs with MAF $>0.05$ and imputation quality $>0.6$ ) and lipids were also undertaken. Details regarding the SNPs and their associated genes utilized in the analyses are provided in Supplementary Table 1.

\section{Statistical methods \\ Comparison of lipids between $A D$ and controls}

Inverse normal transformed residuals for the individual lipids and group lipids were obtained after regressing out the effect of possible confounders: age, sex, BMI, diabetes status, hypertension status, medication status for hypertension and hyperlipidemia, $A P O E$ e4 carrier status, education, and current smoking status. This transformed data was used in all subsequent analyses. $T$-tests were used to compare the mean value of lipids residuals between $\mathrm{AD}$ and controls. We used a significance threshold of 0.05 after False discovery rate (FDR) correction for all comparisons. Fold change (FC) was calculated as the ratio of the average lipids abundance in $\mathrm{AD}$ and controls. $T$-tests were also applied to compare lipid abundance between $A \beta$ treated and untreated cells. Between-group comparisons are done assuming unequal variance between groups with an approximation for degrees of freedom.

\section{Classification of $A D$ vs. Control using GLMnet}

We used a machine-learning algorithm, glmnet (elastic net penalization for the generalized linear model [glm]) to classify AD versus control samples. A combination of two penalty functions with two tuning parameters was utilized to shrink the beta coefficients in the $\operatorname{glm}^{21}$. $\mathrm{R}$ (version 3.5.1) $)^{22}$ package caret $^{23}$ for fitting the elastic net glm model with default options was used to identify the optimum values for the tuning parameters.

For classification analysis, the data were randomly split into $70 \%$ training and $30 \%$ test samples maintaining the proportion of cases and controls in the training and test samples as in the full dataset. For the training data, the algorithms were run with three cross-validations with five repeats. To avoid bias due to a single random split of the original data, we have repeated the analysis 10 times and the results were summarized over the 10 iterations.

The glmnet classification accuracy was examined based on several subgroups of the lipid species. The receiver operating curve (ROC) and area under ROC (AUC) were obtained using the R package pROC ${ }^{24}$. Average sensitivity (proportion of $\mathrm{AD}$ cases predicted by the model in the test data), specificity (proportion of controls predicted by the model in the test data), and the AUC across 10 iterations are reported.

\section{Genetic variation and lipid profiles among $A D$ vs. controls}

Linear regression was used to examine the association of $\mathrm{AD}$ risk variants and $\mathrm{AD}$ PRS with the lipids. The inverse normal transformed lipid residuals were used as the dependent variable and individual SNPs or PRS, casecontrols $(\mathrm{CC})$ status (AD vs. controls), and the relevant interaction term $(\mathrm{CC} \times \mathrm{SNP}$ or $\mathrm{CC} \times \mathrm{PRS})$ were used as independent variables. Differential association of the AD risk variants and the PRS with lipids among cases and controls were examined based on the significance of the interaction term.

\section{Results \\ Descriptive statistics}

The sample comprised 82 plasma samples (40 AD patients and 42 cognitively normal 'healthy' controls) from the Sydney MAS cohort. The demographic information of participants is displayed in Table 1 . The AD patients were significantly older than the controls with all aged more than 75 years old. There were no gender differences between the 2 groups $\left(\chi^{2}=1.233, p=0.267\right)$. As expected, cognitive scores on the Mini-Mental State Examination (MMSE) were significantly lower in AD patients. Education levels, which represent a protective factor for $\mathrm{AD}$ were measured in years and showed no statistical difference between the $\mathrm{AD}$ and control groups. $\mathrm{AD}$ and controls did not differ in vascular risk factors, including frequency of hypertension, diabetes, and current smoking status. Carriers of the $A P O E^{*} 4$ allele, a strong genetic risk factor for $\mathrm{AD}$ risk, were more common in $\mathrm{AD}$ patients than controls, although this did not reach statistical significance (AD $37.5 \%$ vs. control $19 \%, \chi^{2}=3.457, p=0.063$ ). The elevated levels of oligomeric $\mathrm{A} \beta$ in the plasma distinguished the $\mathrm{AD}$ and control groups and were associated with increased MMSE, in patients with AD.

\section{Comparison of lipids between AD and control patients}

A total of 778 distinct lipid species from 9 lipid classes were analyzed, including neutral lipids such as 14 cholesteryl esters (ChE), 50 diglycerides (DG), 382 triglycerides (TG); sphingolipids including 43 ceramides (Cer), 90 sphingomyelins (SM); and phospholipid subclasses including 120 phosphatidylcholines (PC), 24 lyso- 
Table 1 Characteristics of Alzheimer's disease and control participants.

\begin{tabular}{llll}
\hline & Healthy controls $(\boldsymbol{n}=\mathbf{4 2})$ & Alzheimer's disease $(\boldsymbol{n}=\mathbf{4 0})$ & Difference \\
\hline Age(years) & $81.27(2.48)$ & $86.72(5.03)$ & $t=6.270, p<0.01$ \\
Sex (males/females) & $19 / 23$ & $23 / 17$ & $x^{2}=1.233, p=0.267$ \\
Oligomeric A (OD value) & $0.25(0.05)$ & $0.40(0.10)$ & $t=6.391, p<0.01$ \\
Education (years) & $11.30(3.59)$ & $11.59(3.64)$ & $t=0.363, p=0.717$ \\
Body mass index & $26.69(3.19)$ & $25.45(3.72)$ & $t=1.631, p=0.107$ \\
Diabetes & $7.10 \%$ & $15 \%$ & $x^{2}=1.294, p=0.255$ \\
Hypertension & $61.90 \%$ & $77.50 \%$ & $x^{2}=2.351, p=0.125$ \\
Anti-hypertensive medication & $60.00 \%$ & $54.30 \%$ & $x^{2}=0.249, p=0.618$ \\
Anti-hyperlipidemia medication & $64.30 \%$ & $50.00 \%$ & $x^{2}=1.709, p=0.791$ \\
Current smoker & $9.50 \%$ & $5.00 \%$ & $x^{2}=0.618, p=0.432$ \\
APOE 4 carriers & $19 \%$ & $37.50 \%$ & $x^{2}=3.457, p=0.063$ \\
MMSE & $28.21(1.58)$ & $22.63(3.86)$ & $t=8.557, p<0.01$ \\
\hline
\end{tabular}

Continuous variables are expressed as means and standard deviations. Categorical variables are expressed as $\%$.

$A P O E^{*} 4$ apolipoprotein epsilon 4, MMSE Mini-mental state examination.

phosphatidylcholines(LPC), 38 phosphatidylethanolamines (PE), and 17 phosphatidylinositols (PI). The proportion of lipids significantly different between $\mathrm{AD}$ and controls according to each of the examined lipid classes are shown in Fig. 1. Volcano plots were drawn according to lipid fold changes of abundance comparing $\mathrm{AD}$ and controls and FDR-corrected $p$ values in for all individual lipid species (see Fig. 2 and Supplementary Table 2).

Almost all ceramides were significantly different between control and AD groups after adjustment for age, sex, and vascular risk factors, even after multiple testing corrections. Molecular profiles of the ceramidome showed that dihydroxy Cer species comprising fatty acyls (18C) with 0 or 2 double bonds and all dihydroxy Cer with 19C were significantly higher in $\mathrm{AD}$. On the other hand, dihydroxy Cer containing $16 \mathrm{C}$ or $18 \mathrm{C}$ with 1 double bond showed the opposite results. Most monohydroxy and trihydroxy Cer were significantly lower in $\mathrm{AD}$ subjects. Four AD positive ceramides including Cer(d18:0_16:0), Cer(d19:1_24:0), Cer (d18:0_23:0), and Cer(d18:2_25:0), and AD negatively correlated ceramides: Cer(d18:1_23:0), Cer(t16:1_14:0), Cer(m18:1_20:0), $\operatorname{Cer}\left(\mathrm{m} 18: 0 \_22: 0\right)$, and $\operatorname{Cer}\left(\mathrm{d} 19: 1 \_22: 0\right)$ showed the largest fold changes $(\mathrm{FC}>4)$. Sphingomyelin is another type of sphingolipid. Most lipids in SM class showed significantly lower levels in AD plasma. The lipids with FC $>4$ included $\mathrm{SM}(\mathrm{d} 35: 4), \mathrm{SM}(\mathrm{d} 34: 1), \mathrm{SM}(\mathrm{d} 31: 1)$, $\mathrm{SM}(\mathrm{d} 18: 1$ 21:0), $\mathrm{SM}(\mathrm{t} 36: 2)$, and $\mathrm{SM}(\mathrm{d} 40: 4)$. In contrast, $\mathrm{SM}(\mathrm{d} 41: 1), \mathrm{SM}(\mathrm{d} 41: 4)$, and $\mathrm{SM}\left(\mathrm{d} 18: 1 \_24: 3\right)$ were largest downregulated in $\mathrm{AD}$.

In the $\mathrm{AD}$ cases, there was a higher abundance of $\mathrm{PC}$ lipids compared to controls. They were comprised of very short and short-chain fatty acyls except for PC containing
C15 without double bonds and C16 with 2 double bonds. The largest fold changes were observed in $\mathrm{PC}\left(16: 0 \_22: 6\right)$, PC(18:2_18:2), PC(18:0_20:4), PC(36:2), PC(16:1_22:5) that showed upregulation in AD. PC (20:2_18:2) and PC (38:7) showed lower plasma concentrations in AD. LPCs with longer fatty acid chains tended to be lower in $A D$ patients. No LPC lipids differed significantly between cognitively normal and AD groups. Most of PE lipids were lower in AD-affected patients. Major PE lipids were reduced in $\mathrm{AD}$, including highly changed $(\mathrm{FC}>4) \mathrm{PE}$ (16:0p_22:6), PE(18:0p_20:4), PE(16:0p_18:1), and PE (18:0p_22:4). PE (18:0_18:1) was higher in plasma of Alzheimer's patients. PI showed an elevated abundance in $\mathrm{AD}$ and the PI lipids, with PI (18:0_18:3) and PI (18:1_20:4) the most significantly elevated in AD cases.

ChE (18:3), ChE (20:3), and ChE (22:3) showed the largest fold changes. Consistent with the result of group DGs, most DG lipids species were higher in AD patients, including DG(16:0_18:3), DG(22:4e), DG(17:1_18:1), DG (20:0_18:2), and DG(36:4e) with largest fold changes. Most downregulated DGs include DG(18:1_20:4), DG (16:0_18:1), and DG(18:0_18:1).

As well, around $30 \%$ of TG lipids were observed to be higher in $\mathrm{AD}$ patients. The most upregulated TG lipids include TG(18:1_17:1_18:3), TG(14:0_18:2_20:5), TG (16:1_20:1_22:4), TG(60:10), and TG(18:1_18:1_22:5). On the other hand, most of the long-chain polyunsaturated fatty acid-containing TGs were reduced in $\mathrm{AD}$ compared to agematched controls. About 18\% of plasma TG lipids are decreased in $\mathrm{AD}$ patients, and TG(18:1_12:0_14:0), TG (18:4_16:1_18:3), TG(16:0_20:4_22:6), TG(16:0_14:0_18:1), and TG(16:0_16:0_16:0) showed the largest fold changes. We 


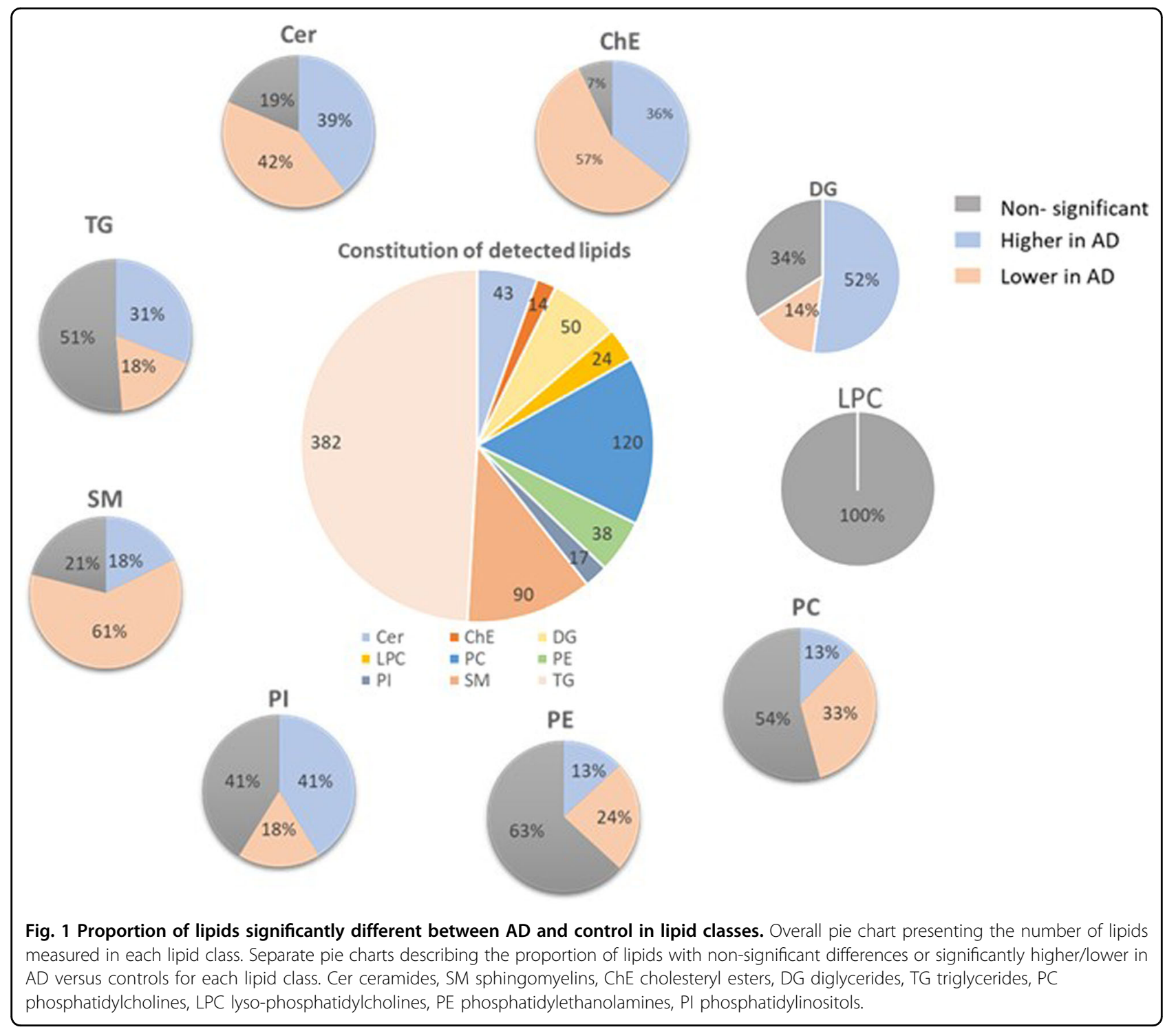

also identified suggestive group differences between almost all species containing more than 2 double bonds. Nine lipid groups calculated by summarizing all lipid species in the same lipid class were compared between $\mathrm{AD}$ and controls (Fig. 3 and Supplementary Table 3). Only the DG group was significantly higher in $\mathrm{AD}(p=0.009$, mean of residuals: Control: $-0.276 \pm 0.143, \quad \mathrm{AD}: 0.290 \pm 0.154)$. Other lipid group's lipids did not differ after adjusting for covariates.

\section{Cell lipidomics}

Results are plotted in Fig. 4. The concentration of oligomeric peptide used in this study is similar to that reported from human CSF and human cell culture conditioned medium $^{25,26}$. Additionally, it also represents a concentration closer to the levels found in brain ${ }^{25,26}$ offering a more physiologic impression of the effect of $A \beta$ oligomers on astrocyte glial lipid profiles. SMs were significantly elevated in astroglioma cells treated with $A \beta_{42}$ oligomers compared to nontreated cells $(p=0.0015)$. No significant differences were found in phospholipids except that the level of the PI group was higher in the $\mathrm{A} \beta_{42}$ group $(p=0.041)$, which is consistent with the result found in human $\mathrm{AD}$ plasma samples. Even though PE and LPE were not significantly different between treated and non-treated cells, the ratio of LPE/PE significantly increased in $\mathrm{A} \beta_{42}$-treated astrogliomas ( $p$ value). TG was also increased in the $\mathrm{A} \beta_{42}$ group $(p=0.005)$.

\section{Classification of $A D$ vs. Control using lipid profiles}

Several models were used for the classification of AD versus controls based on the plasma lipidome profile. The glmnet package was used in all the analyses. The analyses were repeated 10 times and results were summarized 


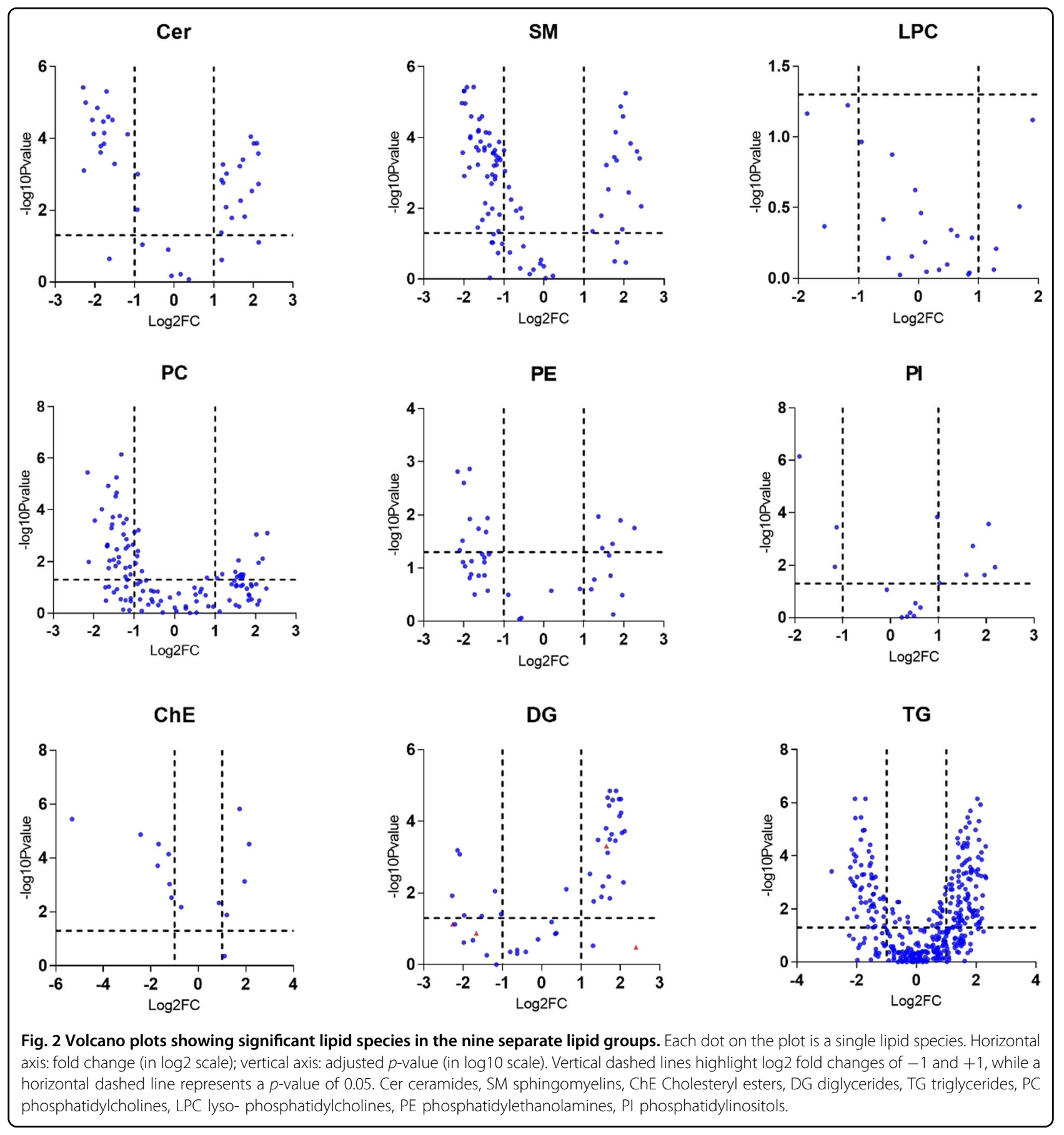

across the 10 iterations. The algorithms were run in turn using the full list of individual lipids, all of the 9 subclasses of lipids, and group lipids. The average sensitivity, specificity, and AUC of the test data are summarized in Table 2. The ROC curves for the top 3 models, ChE, SM, and TG are presented in Fig. 5 and resulted in good classification accuracy with more than $80 \%$ AUC. In general, group lipids and the lipid subclasses LPC and PE had less classification accuracy compared to the other subclasses.

\section{SNPs and lipids with $A D$ versus control}

The associations of AD PRS and AD-related SNPs with the lipidome were examined. The individual lipids that were significantly different between $\mathrm{AD}$ and controls $(n=420)$ were utilized to explore the association. No significant association of AD PRS with lipids was observed after FDR correction (Supplementary Table 4). However, the AD PRS $\times$ CC interaction was nominally significant $(p$-value $<$ $=0.05$ ) for 57 lipid species (Table 3 ), which were mainly 


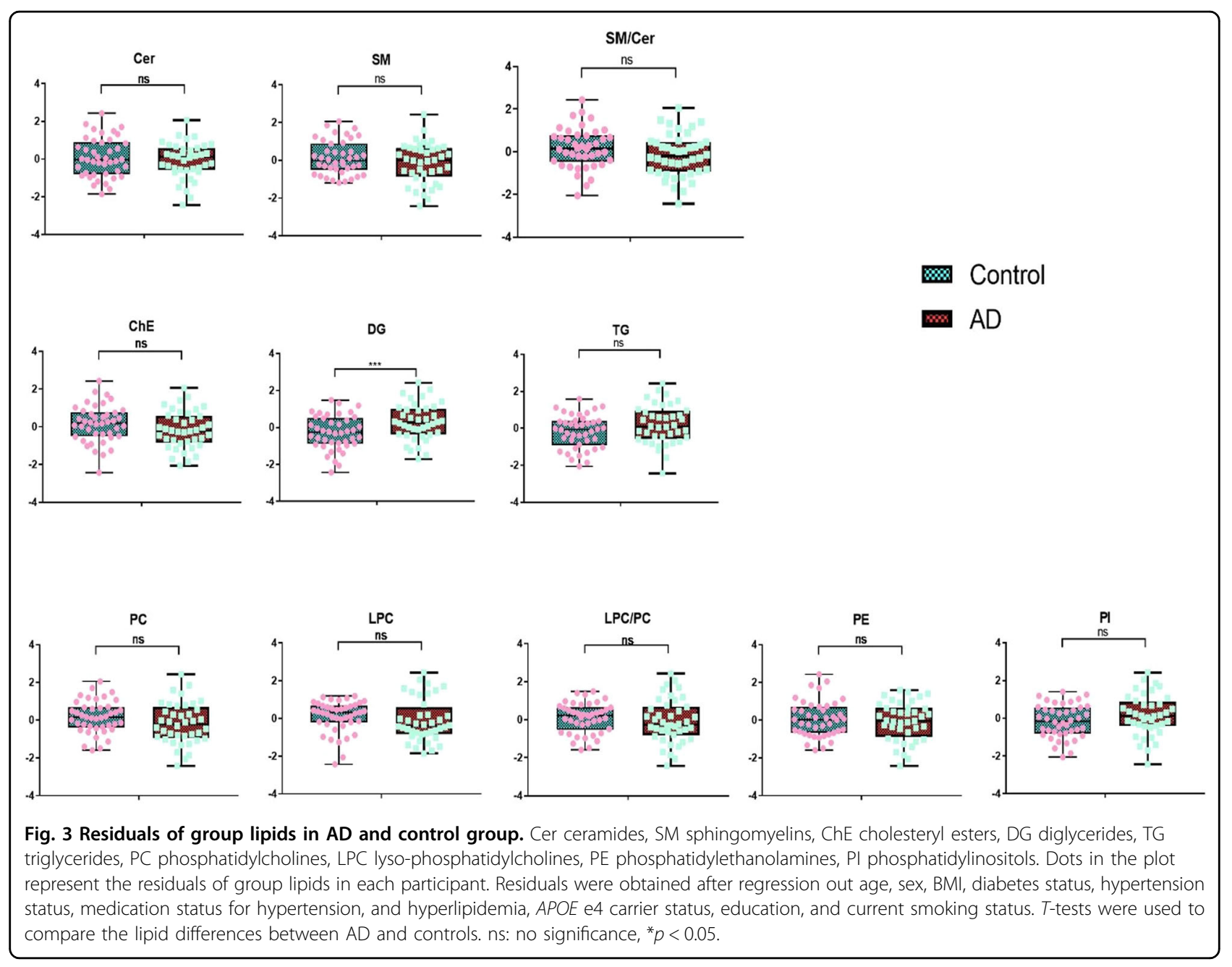

PCs, SMs, and TGs. Out of the 57 significant interactions, only two PRS scores had marginal significant effects $(p$ value $<0.05)$. The same analysis was also repeated using 33 AD implicated individual SNPs recently identified in another study ${ }^{20}$. All of these individual SNPs were found to have different effects on a certain number of lipids between $\mathrm{AD}$ and controls before adjustment for multiple testing. Nominally significantly different associations of SNPs with lipids between $\mathrm{AD}$ and controls are listed in Supplementary Table 5.

We further explored the association of AD-associated genes $(n=19)$ derived from the list of 33 SNPs with lipids in each lipid class (Table 4). FERMT2 and $M S 4 A 6 A$ showed a significantly differential association with lipids in all lipid classes across disease and control groups. In addition, PC, SM, and TG lipids were differentially associated with the largest number of ADrelated genes. The heatmap in Fig. 6 shows the proportion of lipids in each lipid class having differential associations with the $\mathrm{AD}$-associated genes. $A B C A 7$ had a differential association with more than half of the DG lipids (52.63\%) and PI lipids (57.14\%), respectively. Additionally, $43.4 \%$ of lipids in the SM class were differentially associated with $C L U$. More than $30 \%$ of lipids in ChE, PE, and TG classes had differential associations with separate genes (ChE-PICALM, SLC24A4, and SORL1; PE-CLU and CR1; TG-BINI) between AD and control group. Finally, we found 43 lipids that were significantly associated with both the AD-related genes and PRS (Table 5).

\section{Discussion}

In this study, significant differences in plasma lipids between $\mathrm{AD}$ cases and controls were observed. In vitro analyses demonstrated similar changes in astroglial cells when treated with $A \beta_{42}$. GLMnet algorithm was used to distinguish $\mathrm{AD}$ from controls using different plasma lipidbased models. SMs, ChEs, and TGs showed the greatest accuracy in discriminant analysis with AUC of more than $80 \%$. Finally, associations between AD case-control status and genetic risk factors for $\mathrm{AD}$ were nominally significant when examining lipid profiles. 


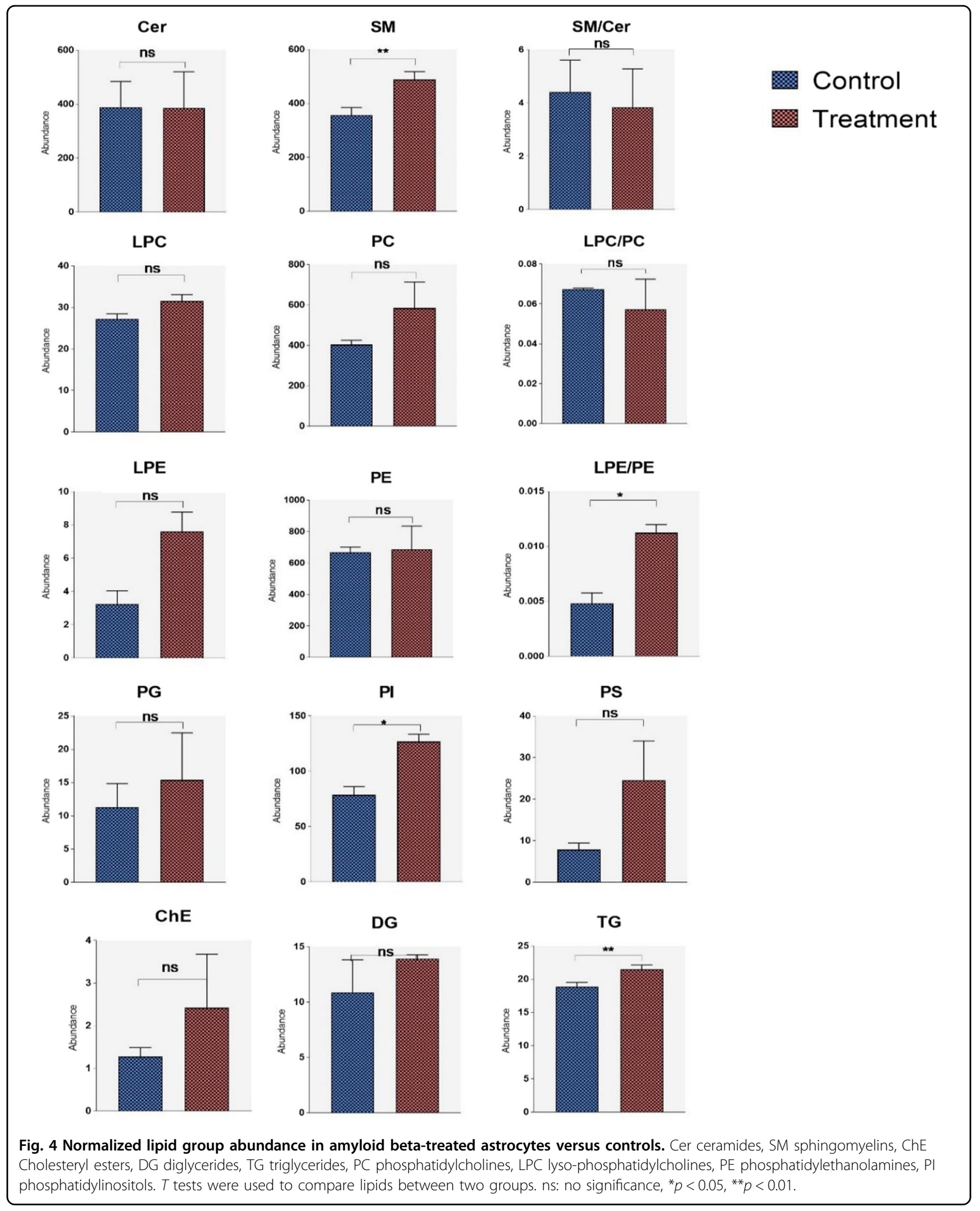


Table 2 GLMnet results of lipid models in the classification of $A D$ versus control.

\begin{tabular}{llll}
\hline Lipid models & Sensitivity & Specificity & AUC \\
\hline All_lipids & 0.66 & 0.73 & 0.78 \\
Cer & 0.7 & 0.79 & 0.79 \\
ChE & 0.78 & 0.75 & 0.82 \\
DG & 0.72 & 0.69 & 0.76 \\
LPC & 0.49 & 0.62 & 0.58 \\
PC & 0.69 & 0.76 & 0.78 \\
PE & 0.58 & 0.5 & 0.57 \\
PI & 0.7 & 0.76 & 0.79 \\
SM & 0.71 & 0.78 & 0.83 \\
TG & 0.65 & 0.82 & 0.83 \\
SM_TG & 0.69 & 0.75 & 0.81 \\
ChE_SM & 0.7 & 0.81 & 0.79 \\
ChE_TG & 0.71 & 0.78 & 0.82 \\
ChE_SM_TG & 0.67 & 0.77 & 0.79 \\
\hline
\end{tabular}

Cer ceramides, SM sphingomyelins, ChE cholesteryl esters, DG diglycerides, $T G$ triglycerides, $P C$ phosphatidylcholines, LPC lyso-phosphatidylcholines, $P E$ phosphatidylethanolamines, PI phosphatidylinositols, GLMnet elastic net penalization for the generalized linear model.

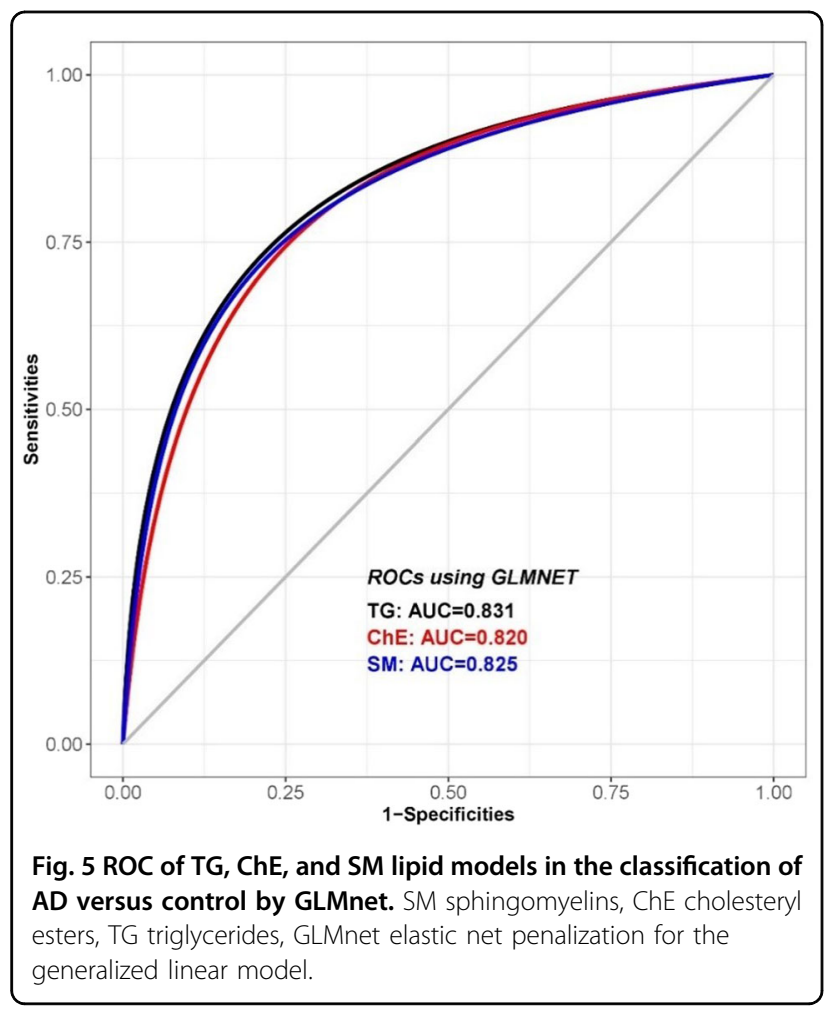

\section{Identification of the roles of lipids associated with $A D$}

Our study did not show a significant group ceramide difference between AD and controls. However, we found that most individual ceramides differed significantly between these two groups. Molecular profiles of the ceramidome showed that dihydroxy Cer species, comprising fatty acyls (18C) with 0 or 2 double bonds and all dihydroxy Cers with 19C, were significantly increased in AD. On the other side, dihydroxy Cers containing $16 \mathrm{C}$ or $18 \mathrm{C}$ with 1 double bond showed an opposite result. Most monohydroxy and trihydroxy Cers decreased significantly in $\mathrm{AD}$ subjects, which might suggest the opposite pathobiology of different Cer subgroups. Ceramides are essential for the maintenance of membrane structure stabilization, cell-to-cell recognition, and secondary messenger signaling ${ }^{27}$. Dysregulation of Cers has been linked to cognitive decline and brain atrophy ${ }^{28}$. Higher levels of serum ceramide were associated with increased risk of $\mathrm{AD}$ compared to subjects with the lowest tertile of serum ceramide levels in another study ${ }^{29}$. In addition, even though the sum of SM lipids was not significantly different between the two groups, we found that most individual SM lipids were lower in AD plasma. Altered sphingolipid metabolism has been associated with the pathogenesis of several neurodegenerative diseases including $\mathrm{AD}$, and metabolic syndrome ${ }^{29-32}$. The decrease in SM lipids has been shown to be strongly correlated with parameters of insulin resistance, and lipid metabolism, which are major risk factors for $\mathrm{AD}^{33}$. A cross-sectional study ( $n=26 \mathrm{NC}, 26 \mathrm{AD})$ observed reduced $\mathrm{SM}$ species in $\mathrm{AD}$ versus controls and long aliphatic chains $(\mathrm{C} 22, \mathrm{C} 24)$ in particular ${ }^{31}$, which is consistent with our results.

We did not find any significant differences among group PC lipids. However, the AD population tended to present higher abundance in individual $\mathrm{PC}$ lipids comprising very short and short-chain fatty acyls except for PC containing C15 without double bonds and C16 with 2 double bonds. Recent studies have demonstrated altered PC metabolism in cognitively impaired elderly and $\mathrm{AD}$ patients ${ }^{9,34,35}$. Similar to PC lipids, we did not observe significant changes in group PE. About 24\% of individual PEs were all lower in $\mathrm{AD}$ patients, which is almost 2 fold of PEs that were higher in $\mathrm{AD}$. $\mathrm{PE}$ has been previously reported to be significantly reduced in the brains of individuals with $\mathrm{AD}$ and $\mathrm{HD}^{36}$. Since a multitude of neuropathological processes can lead to a decrease in PC and PE, it is likely that reduced levels of these phospholipids may be linked to the observed neuronal loss. Increased activities of the Kennedy pathway enzymes, phosphoethanolamine cytidylyltransferase, phosphocholine cytidylyltransferase, and PS synthase have been previously reported to be elevated in the diseased brain regions of patients with $\mathrm{AD}$ and $\mathrm{PD}^{34}$. Contrary to the decreased trend of other phospholipids, including PC and PE, we observed an opposite change in PI in AD patients, with most of the individual PI lipids higher in AD subjects. PI is another phospholipid that is 
Table 3 Nominally significantly differential association of the AD risk PRS with lipids between AD and controls control.

\begin{tabular}{|c|c|c|c|c|c|c|c|c|}
\hline Lipid & Beta.PRS & Beta.PRS_CC & SE.PRS & SE.PRS_CC & t.PRS & t.PRS_CC & Pval.PRS & Pval.PRS_CC \\
\hline Cer(d18:1_16:0) & 0.197 & -0.511 & 0.133 & 0.195 & 1.483 & -2.624 & 0.142 & 0.011 \\
\hline Cer(t16:1_14:0) & 0.268 & -0.510 & 0.141 & 0.207 & 1.894 & -2.461 & 0.062 & 0.016 \\
\hline ChE(22:5) & -0.080 & 0.539 & 0.137 & 0.200 & -0.583 & 2.690 & 0.562 & 0.009 \\
\hline PC(16:0_20:4) & -0.107 & 0.495 & 0.144 & 0.211 & -0.745 & 2.345 & 0.459 & 0.022 \\
\hline PC(16:0_20:5) & 0.001 & 0.535 & 0.144 & 0.210 & 0.007 & 2.544 & 0.994 & 0.013 \\
\hline PC(16:0_22:6) & 0.150 & 0.419 & 0.123 & 0.180 & 1.221 & 2.331 & 0.226 & 0.022 \\
\hline$P C\left(16: 2 e \_16: 0\right)$ & 0.089 & -0.428 & 0.145 & 0.212 & 0.612 & -2.018 & 0.543 & 0.047 \\
\hline PC(18:0_20:3) & 0.131 & 0.433 & 0.135 & 0.198 & 0.973 & 2.187 & 0.334 & 0.032 \\
\hline PC(18:1_20:3) & 0.025 & 0.441 & 0.143 & 0.209 & 0.174 & 2.108 & 0.863 & 0.038 \\
\hline PC(18:2_18:2) & -0.005 & 0.415 & 0.138 & 0.202 & -0.039 & 2.053 & 0.969 & 0.044 \\
\hline $\mathrm{PC}(34: 0)$ & 0.057 & -0.510 & 0.142 & 0.208 & 0.404 & -2.447 & 0.688 & 0.017 \\
\hline$P C(37: 2)$ & 0.285 & -0.488 & 0.147 & 0.215 & 1.942 & -2.267 & 0.056 & 0.026 \\
\hline PE(16:0_20:3) & -0.059 & 0.445 & 0.144 & 0.210 & -0.413 & 2.117 & 0.681 & 0.038 \\
\hline PI(18:0_18:2) & 0.026 & -0.421 & 0.123 & 0.180 & 0.208 & -2.346 & 0.836 & 0.022 \\
\hline $\mathrm{PI}(36: 3)$ & -0.163 & 0.603 & 0.148 & 0.217 & -1.105 & 2.784 & 0.273 & 0.007 \\
\hline SM(d17:1_13:0) & 0.353 & -0.668 & 0.142 & 0.209 & 2.476 & -3.200 & 0.016 & 0.002 \\
\hline SM(d17:1_18:3) & 0.041 & -0.405 & 0.134 & 0.196 & 0.303 & -2.064 & 0.763 & 0.043 \\
\hline SM(d18:2_24:3) & 0.240 & -0.470 & 0.145 & 0.213 & 1.653 & -2.207 & 0.103 & 0.030 \\
\hline SM(d32:4) & 0.166 & -0.585 & 0.134 & 0.197 & 1.233 & -2.971 & 0.222 & 0.004 \\
\hline $\operatorname{SM}(d 33: 1)$ & 0.095 & -0.521 & 0.139 & 0.204 & 0.683 & -2.551 & 0.497 & 0.013 \\
\hline SM(d34:2) & 0.132 & -0.416 & 0.141 & 0.206 & 0.933 & -2.014 & 0.354 & 0.048 \\
\hline SM(d35:1) & 0.204 & -0.462 & 0.148 & 0.216 & 1.384 & -2.134 & 0.171 & 0.036 \\
\hline SM(d36:3) & 0.365 & -0.568 & 0.146 & 0.213 & 2.506 & -2.662 & 0.014 & 0.010 \\
\hline $\operatorname{SM}(d 37: 2)$ & -0.012 & -0.429 & 0.136 & 0.199 & -0.090 & -2.161 & 0.929 & 0.034 \\
\hline$S M(d 38: 1)$ & 0.006 & -0.419 & 0.127 & 0.186 & 0.049 & -2.250 & 0.961 & 0.027 \\
\hline $\operatorname{SM}(d 38: 2)$ & 0.027 & -0.458 & 0.137 & 0.200 & 0.197 & -2.285 & 0.844 & 0.025 \\
\hline SM(d39:1) & -0.031 & -0.413 & 0.131 & 0.192 & -0.240 & -2.153 & 0.811 & 0.035 \\
\hline SM(d39:2) & 0.108 & -0.516 & 0.139 & 0.203 & 0.776 & -2.539 & 0.440 & 0.013 \\
\hline $\operatorname{SM}(d 40: 1)$ & 0.038 & -0.453 & 0.135 & 0.198 & 0.283 & -2.294 & 0.778 & 0.025 \\
\hline $\mathrm{SM}(\mathrm{d} 40: 3)$ & 0.118 & -0.458 & 0.149 & 0.218 & 0.789 & -2.098 & 0.433 & 0.039 \\
\hline SM(d41:4) & -0.070 & -0.397 & 0.135 & 0.198 & -0.520 & -2.003 & 0.605 & 0.049 \\
\hline $\operatorname{SM}(\mathrm{d} 42: 1)$ & 0.103 & -0.435 & 0.138 & 0.202 & 0.746 & -2.152 & 0.458 & 0.035 \\
\hline $\mathrm{SM}(\mathrm{d} 42: 2)$ & 0.028 & -0.507 & 0.129 & 0.189 & 0.221 & -2.688 & 0.826 & 0.009 \\
\hline $\mathrm{SM}(\mathrm{d} 42: 3)$ & 0.041 & -0.494 & 0.140 & 0.205 & 0.296 & -2.409 & 0.768 & 0.018 \\
\hline $\operatorname{SM}(\mathrm{d} 43: 1)$ & 0.106 & -0.500 & 0.147 & 0.215 & 0.721 & -2.328 & 0.473 & 0.023 \\
\hline $\operatorname{SM}(\mathrm{d} 43: 2)$ & 0.167 & -0.606 & 0.140 & 0.204 & 1.200 & -2.966 & 0.234 & 0.004 \\
\hline SM(d44:4) & 0.019 & -0.421 & 0.136 & 0.200 & 0.143 & -2.107 & 0.887 & 0.038 \\
\hline$S M(d 44: 5)$ & 0.134 & -0.575 & 0.131 & 0.192 & 1.028 & -3.001 & 0.308 & 0.004 \\
\hline SM(d44:6) & 0.054 & -0.427 & 0.139 & 0.204 & 0.389 & -2.092 & 0.699 & 0.040 \\
\hline
\end{tabular}


Table 3 continued

\begin{tabular}{|c|c|c|c|c|c|c|c|c|}
\hline Lipid & Beta.PRS & Beta.PRS_CC & SE.PRS & SE.PRS_CC & t.PRS & t.PRS_CC & Pval.PRS & Pval.PRS_CC \\
\hline SM(t18:0_24:2) & 0.163 & -0.509 & 0.143 & 0.209 & 1.145 & -2.436 & 0.256 & 0.017 \\
\hline SM(t42:1) & 0.240 & -0.578 & 0.139 & 0.204 & 1.724 & -2.832 & 0.089 & 0.006 \\
\hline TG(12:0_17:1_18:2) & -0.122 & 0.473 & 0.144 & 0.211 & -0.848 & 2.243 & 0.399 & 0.028 \\
\hline TG(12:0_18:2_18:2) & -0.159 & 0.555 & 0.134 & 0.196 & -1.191 & 2.832 & 0.237 & 0.006 \\
\hline TG(14:0_14:3_18:2) & -0.136 & 0.470 & 0.143 & 0.209 & -0.950 & 2.250 & 0.345 & 0.027 \\
\hline TG(14:0_18:3_18:3) & -0.046 & 0.518 & 0.135 & 0.197 & -0.345 & 2.623 & 0.731 & 0.011 \\
\hline TG(15:0_14:1_16:1) & -0.048 & 0.463 & 0.129 & 0.189 & -0.375 & 2.453 & 0.709 & 0.017 \\
\hline TG(15:0_16:0_20:5) & -0.063 & 0.480 & 0.133 & 0.195 & -0.474 & 2.464 & 0.637 & 0.016 \\
\hline TG(16:0_14:0_18:3) & -0.142 & 0.468 & 0.138 & 0.202 & -1.032 & 2.317 & 0.305 & 0.023 \\
\hline TG(16:0_14:1_16:1) & -0.198 & 0.579 & 0.146 & 0.213 & -1.360 & 2.711 & 0.178 & 0.008 \\
\hline TG(16:1_12:0_18:2) & -0.046 & 0.421 & 0.142 & 0.208 & -0.324 & 2.023 & 0.747 & 0.047 \\
\hline TG(16:1_20:5_20:5) & 0.088 & 0.503 & 0.126 & 0.185 & 0.696 & 2.724 & 0.488 & 0.008 \\
\hline TG(18:3_14:1_18:2) & -0.045 & 0.412 & 0.141 & 0.206 & -0.319 & 1.999 & 0.751 & 0.049 \\
\hline TG(18:4_14:0_16:1) & -0.155 & 0.616 & 0.143 & 0.209 & -1.082 & 2.944 & 0.283 & 0.004 \\
\hline TG(18:4_16:0_20:4) & -0.081 & 0.525 & 0.141 & 0.207 & -0.571 & 2.533 & 0.570 & 0.013 \\
\hline TG(20:1_20:4_20:4) & 0.304 & -0.531 & 0.144 & 0.210 & 2.117 & -2.524 & 0.038 & 0.014 \\
\hline TG(22:5_18:2_18:2) & -0.078 & 0.454 & 0.147 & 0.215 & -0.529 & 2.110 & 0.598 & 0.038 \\
\hline $\mathrm{TG}(33: 4 \mathrm{e})$ & -0.160 & 0.651 & 0.137 & 0.201 & -1.168 & 3.241 & 0.247 & 0.002 \\
\hline
\end{tabular}

Marginal effect: Beta.PRS/SE.PRS/t.PRS/Pval. PRS: Beta coefficient/standard error/t statistics/p value of association between PRS scores and individual lipids. Interaction effect: Beta.PRS_CC/SE.PRS_CC/t.PRS_CC/Pval.PRS_CC: Beta coefficient/standard error/t statistics/p value of differential association of PRS with lipids between $\mathrm{AD}$ and controls control.

Cer ceramides, SM sphingomyelins, ChE cholesteryl esters, DG diglycerides, TG triglycerides, PC phosphatidylcholines, $L P C$ lyso-phosphatidylcholines, $P E$ phosphatidylethanolamines, PI phosphatidylinositols.

present in the membrane of almost all cell types and is involved in mediating $\mathrm{Ca}^{2+}$ mobilization in response to many hormones, neurotransmitters, and growth factors ${ }^{37}$. Consistently, an animal study showed that lower levels of plasma PC and higher plasma PI were correlated with post-stroke cognitive impairment ${ }^{38}$.

We also found significant differences in ChEs between $\mathrm{AD}$ and controls. ChE is produced in the plasma by the conversion of fatty acids to cholesterol from PC by the enzymatic activity of cholesterol acyl transferase $(\mathrm{LCAT})^{9,39}$. While free cholesterol can be taken up by APOE containing liposomes (e.g. HDL) and is bound to the outer particle surface, esterification enhances cholesterol uptake within the interior of the lipoproteins and enhances cholesterol transport through the bloodstream. LCAT has a preference for highly unsaturated fatty acid chain PC and can link the reduction in PC to dysregulation of specific steps in cholesterol metabolism in $\mathrm{AD}^{40}$. Another enzyme, acyl-coenzyme A can also esterify cholesterol in other tissues. Inhibition of this enzyme has been reported to reduce amyloid plaque load in the brain of $\mathrm{AD}$ mice and improves cognitive outcomes ${ }^{41}$. Consistent with our results showing a significant increase of
DGs in Alzheimer's disease, previous studies have demonstrated augmented levels of DGs in the frontal cortex and plasma of AD patients ${ }^{42}$. DGs are important in maintaining structural integrity and signal transduction ${ }^{42}$ The conversion of DGs to phosphatidic acid by DG kinase has been reported to be decreased in the AD brain ${ }^{43}$. We also found almost $2 / 3$ plasma TG lipids in all significant TG lipids were higher in AD subjects, even though the abundance of group TGs were not different among disease groups. A longitudinal finding was reported in the Honolulu-Asia Aging Study in which a 1SD increase in TG levels during midlife significantly increased the risk of dementia a quarter of a decade later ${ }^{44}$. The exploration of the exact pathobiology of individual TG lipids is needed in the future.

\section{Relationship between plasma lipid profiles and the cell lipidome exposed to oligomeric $A \beta_{42}$ in vivo}

Several studies have reported significant differences in lipid profiles between plasma and postmortem brain tissue $^{45}$. The separation of the brain from the periphery is a major challenge when examining the biological significance of plasma lipids in Alzheimer's disease since not 


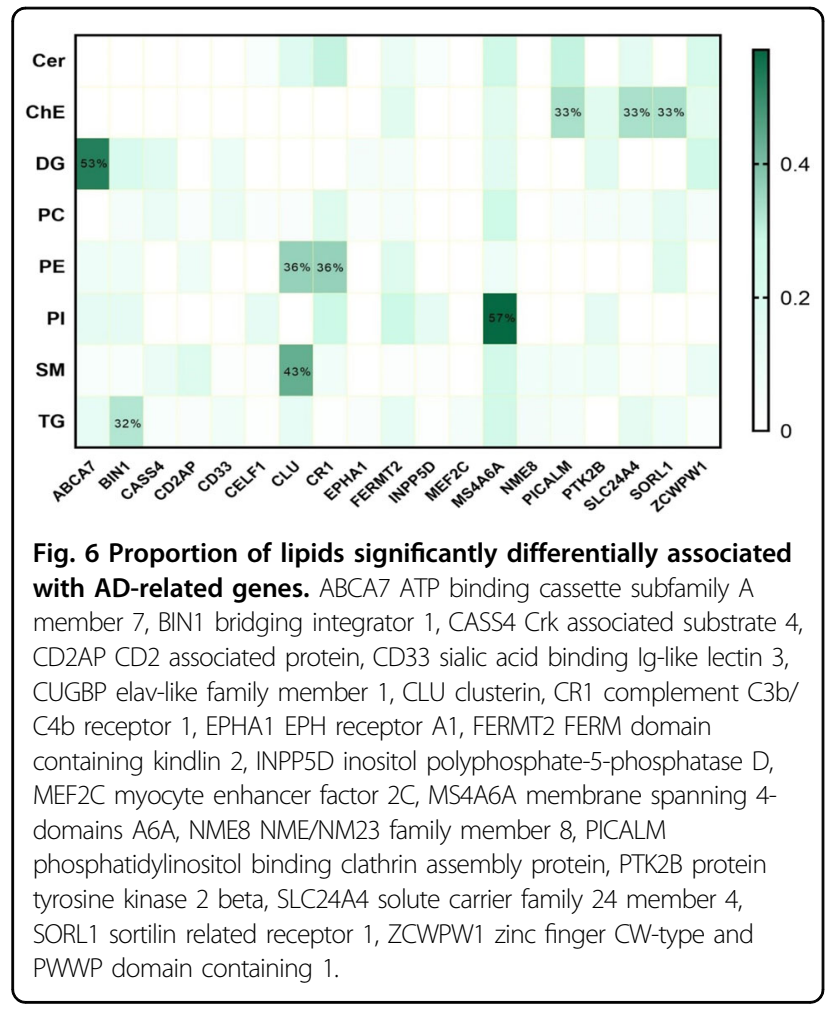

all lipids can be transported across the blood-brain barrier. This separation may likely be compromised by agerelated changes in blood-brain barrier integrity, affecting lipid and $A \beta$ kinetics. To determine whether our observed changes in the plasma lipidome were related to $\mathrm{AD}$ pathology we also quantified the cellular lipidome in human U251 astroglioma cell lines exposed to pathophysiological concentrations of oligomeric $A \beta_{42}$.

Astrocytes are important cells in the CNS and are involved in various physiological aspects ${ }^{46}$. Neurons are thought to be the main source of $A \beta$ in the adult brain. Moreover, the ability of neurons to generate cholesterol in the adult brain is impaired during development. However, astrocytes can produce cholesterol and transport it to neurons with apolipoprotein $\mathrm{E}(\mathrm{apoE})^{47}$. ApoE is the major genetic risk factor for sporadic $\mathrm{AD}$. This has led to the hypothesis that apoE regulates $A \beta$ formation via modulation of lipid raft functions ${ }^{48}$. Recently, astrocytederived cholesterol has been shown to regulate $A \beta$ formation in vivo and influence processes involved in inflammation and neurodegeneration ${ }^{49}$. In our study, we quantified the sum of lipid classes when the astroglioma cell line was exposed to subpathological amounts of oligomeric $A \beta_{42}$. We still found a similar trend of lipid changes between plasma and cells treated with oligomeric $\mathrm{A} \beta_{42}$. For example, SM, PI, and TG were significantly higher oligomeric $A \beta_{42}$-treated cells, and some SM, PI, and TG species were upregulated in AD plasma. However,
Table 4 Differential interaction of genes with lipids between AD and control.

\begin{tabular}{|c|c|c|c|c|c|c|c|c|}
\hline Genes & Cer & ChE & DG & PC & PE & PI & SM & TG \\
\hline$A B C A 7$ & & & $\checkmark$ & & $\checkmark$ & $\checkmark$ & $\checkmark$ & $\checkmark$ \\
\hline BIN1 & & & $\checkmark$ & $\checkmark$ & $\checkmark$ & $\checkmark$ & $\checkmark$ & $\checkmark$ \\
\hline CASS4 & & & $\checkmark$ & $\checkmark$ & & & $\checkmark$ & $\checkmark$ \\
\hline$C D 2 A P$ & & & & $\checkmark$ & $\checkmark$ & & $\checkmark$ & $\checkmark$ \\
\hline CD33 & & & $\checkmark$ & $\checkmark$ & & & $\checkmark$ & $\checkmark$ \\
\hline CELF1 & $\checkmark$ & & & $\checkmark$ & & $\checkmark$ & $\checkmark$ & $\checkmark$ \\
\hline$C L U$ & $\checkmark$ & & & $\checkmark$ & $\checkmark$ & & $\checkmark$ & $\checkmark$ \\
\hline$C R 1$ & $\checkmark$ & & & $\checkmark$ & $\checkmark$ & $\checkmark$ & $\checkmark$ & $\checkmark$ \\
\hline EPHAI & & & $\checkmark$ & $\checkmark$ & & & & $\checkmark$ \\
\hline FERMT2 & $\checkmark$ & $\checkmark$ & $\checkmark$ & $\checkmark$ & $\checkmark$ & $\checkmark$ & $\checkmark$ & $\checkmark$ \\
\hline INPP5D & $\checkmark$ & & & & & $\checkmark$ & $\checkmark$ & $\checkmark$ \\
\hline MEF2C & & & & & & & & $\checkmark$ \\
\hline MS4A6A & $\checkmark$ & $\checkmark$ & $\checkmark$ & $\checkmark$ & $\checkmark$ & $\checkmark$ & $\checkmark$ & $\checkmark$ \\
\hline NME8 & & & & & & & $\checkmark$ & $\checkmark$ \\
\hline PICALM & $\checkmark$ & $\checkmark$ & & $\checkmark$ & & & $\checkmark$ & $\checkmark$ \\
\hline PTK2B & & $\checkmark$ & $\checkmark$ & $\checkmark$ & & $\checkmark$ & $\checkmark$ & \\
\hline SLC24A4 & $\checkmark$ & $\checkmark$ & & $\checkmark$ & & & $\checkmark$ & $\checkmark$ \\
\hline SORLI & & $\checkmark$ & & $\checkmark$ & $\checkmark$ & & $\checkmark$ & $\checkmark$ \\
\hline ZCWPW1 & $\checkmark$ & $\checkmark$ & $\checkmark$ & $\checkmark$ & & & $\checkmark$ & $\checkmark$ \\
\hline
\end{tabular}

" $\checkmark$ ": $\geq 1$ lipid in each lipid class were differentially associated with the named gene between $A D$ and controls.

Cer ceramides, SM sphingomyelins, ChE cholesteryl esters, DG diglycerides, TG triglycerides, $P C$ phosphatidylcholines, $L P C$ lyso-phosphatidylcholines, $P E$ phosphatidylethanolamines, $\mathrm{PI}$ phosphatidylinositols.

no significant differences were observed between $\mathrm{AD}$ and control in plasma and cells for other lipid groups. The results in the cellular lipidome support findings that $A \beta_{42}$ plays a contributory if not causal role in mediating changes in plasma and cellular lipid profiles in AD.

\section{The lipidome signature as a biomarker of $A D$}

We also examined whether a battery of plasma lipids can be used to discriminate AD patients from controls. All individual lipids, 9 subgroup lipids, and group lipids were used in the GLMnet algorithm. We observed that three classification models, using either TG, ChE, and SM, had significant power in discriminating $\mathrm{AD}$ from controls, with $>80 \%$ AUC. Additionally, individual lipids in TG, $\mathrm{ChE}$, and SM showed even higher AUC than group lipids. Therefore, measurement of these group lipids using mass spectrometry will provide renewed insight in the underlying mechanisms of $\mathrm{AD}$ and provide additional drug targets. Taken together, our findings suggest that alterations in both cholesterol and sphingolipid metabolism may play an important role in the pathobiology of $\mathrm{AD}$. 
Table 5 Overlap of lipids with significant association with SNPs and PRS scores.

\begin{tabular}{|c|c|c|c|c|}
\hline Lipid & Number of associated SNPs & Most significant Pval.SNPs & Gene (minimum Pvalue.SNPs) & Pval.PRS_CC \\
\hline Cer(d18:1_16:0) & 3 & 0.009 & $C L U$ & 0.011 \\
\hline $\operatorname{ChE}(22: 5)$ & 5 & 0.014 & SORL1 & 0.009 \\
\hline PC(16:0_20:4) & 1 & 0.045 & CASS4 & 0.022 \\
\hline PC(16:0_20:5) & 2 & 0.033 & SORL 1 & 0.013 \\
\hline PC(18:0_20:3) & 1 & 0.027 & MS4A6A & 0.032 \\
\hline PC(18:1_20:3) & 1 & 0.042 & CELF1 & 0.022 \\
\hline PC(18:2_18:2) & 1 & 0.007 & FERMT2 & 0.044 \\
\hline PE(16:0_20:3) & 1 & 0.005 & CR1 & 0.038 \\
\hline $\operatorname{Pl}(36: 3)$ & 1 & 0.002 & INPP5D & 0.007 \\
\hline SM(d17:1_13:0) & 2 & 0.014 & SORL 1 & 0.002 \\
\hline SM(d17:1_18:3) & 3 & 0.040 & $C L U$ & 0.043 \\
\hline $\operatorname{SM}(d 32: 4)$ & 3 & 0.008 & MS4A6A & 0.004 \\
\hline $\operatorname{SM}(d 33: 1)$ & 5 & 0.021 & $C L U$ & 0.013 \\
\hline $\mathrm{SM}(\mathrm{d} 34: 2)$ & 2 & 0.045 & CLU & 0.048 \\
\hline $\operatorname{SM}(d 35: 1)$ & 1 & 0.012 & MS4A6A & 0.036 \\
\hline $\operatorname{SM}(d 37: 2)$ & 4 & 0.014 & CD2AP & 0.034 \\
\hline $\operatorname{SM}(d 38: 1)$ & 3 & 0.041 & CLU & 0.027 \\
\hline SM(d38:2) & 3 & 0.022 & CLU & 0.025 \\
\hline SM(d39:1) & 3 & 0.027 & $C L U$ & 0.035 \\
\hline $\operatorname{SM}(d 39: 2)$ & 3 & 0.027 & $C L U$ & 0.013 \\
\hline $\operatorname{SM}(d 40: 1)$ & 5 & 0.021 & $C L U$ & 0.025 \\
\hline$S M(d 41: 4)$ & 1 & 0.028 & MS4A6A & 0.049 \\
\hline $\mathrm{SM}(\mathrm{d} 42: 1)$ & 2 & 0.013 & MS4A6A & 0.035 \\
\hline $\operatorname{SM}(\mathrm{d} 42: 2)$ & 5 & 0.012 & MS4A6A & 0.009 \\
\hline $\operatorname{SM}(\mathrm{d} 42: 3)$ & 3 & 0.044 & $A B C A 7$ & 0.018 \\
\hline $\operatorname{SM}(\mathrm{d} 43: 1)$ & 2 & 0.030 & $C D 2 A P$ & 0.023 \\
\hline $\mathrm{SM}(\mathrm{d} 43: 2)$ & 1 & 0.032 & CASS4 & 0.004 \\
\hline $\mathrm{SM}(\mathrm{d} 44: 4)$ & 3 & 0.016 & $C L U$ & 0.038 \\
\hline$S M(d 44: 5)$ & 4 & 0.026 & $C L U$ & 0.004 \\
\hline SM(t18:0_24:2) & 3 & 0.022 & MS4A6A & 0.017 \\
\hline SM(t42:1) & 5 & 0.023 & MS4A6A & 0.006 \\
\hline TG(12:0_17:1_18:2) & 1 & 0.013 & NME8 & 0.028 \\
\hline TG(14:0_14:3_18:2) & 1 & 0.037 & BIN1 & 0.027 \\
\hline TG(14:0_18:3_18:3) & 3 & 0.003 & NME8 & 0.011 \\
\hline TG(15:0_14:1_16:1) & 1 & 0.032 & NME8 & 0.017 \\
\hline TG(15:0_16:0_20:5) & 2 & 0.048 & BIN1 & 0.016 \\
\hline TG(16:0_14:0_18:3) & 5 & 0.011 & $C L U$ & 0.023 \\
\hline TG(16:0_14:1_16:1) & 1 & 0.018 & SORL 1 & 0.008 \\
\hline TG(18:3_14:1_18:2) & 2 & 0.035 & $A B C A 7$ & 0.049 \\
\hline
\end{tabular}


Table 5 continued

\begin{tabular}{lllll}
\hline Lipid & Number of associated SNPs & Most significant Pval.SNPs & Gene (minimum Pvalue.SNPs) & Pval.PRS_CC \\
\hline TG(18:4_14:0_16:1) & 1 & 0.045 & ZCWPW1 & 0.004 \\
TG(18:4_16:0_20:4) & 3 & 0.011 & CLU & 0.013 \\
TG(22:5_18:2_18:2) & 4 & 0.017 & NME8 & 0.038 \\
TG(33:4e) & 4 & 0.011 & MS4A6A & 0.002 \\
\hline
\end{tabular}

Only lipids with significant differential associations with both PRS and SNPs were included in the table. The most significant $p$ value of lipids association with SNPs of the same gene was presented when the SNP had the most significant differential association with the lipid across AD and control.

\section{$A D$ genetic risk, lipids, and $A D$}

We examined the relationships between AD PRS and the AD-associated lipids in our sample. Even though no significant association was found after FDR correction, we observed a number of lipid species that were nominally differentially affected between $\mathrm{AD}$ and controls, most of which were PC, SM, and TGs. Our data suggest that these lipids might mediate the effect of several SNPs linked to AD.

We also investigated individual AD-related SNPs and lipid levels. Significant associations between AD-related SNPs and individual lipids were only observed before FDR correction. However, we found all SNPs having nominally significant differential association with a certain number of lipid species between AD and control group.

FERMT2 and MS4A6A genes showed a significantly differential association with lipids in all lipid classes across disease groups. FERMT2 is a member of the Fermitin family of proteins, which are involved in cell-matrix adhesion complexes. Shulman et al. ${ }^{50}$ validated the association of FERMT2 with $\mathrm{AD}$ risk after performing a gene screen and in vivo studies in Drosophila melanogaster. Their work showed altered expression of both FERMT2 and CELF1 homologs modulates tau neurotoxicity. It is also upregulated in atherosclerotic plaques, which is a risk factor for $\mathrm{AD}^{51}$. The correlation of FERMT2 with poststroke brain recovery ${ }^{52}$ and higher plasma PI in poststroke mice $^{38}$ supports the relationship between FERMT2 and PI in our study, i.e. the PI class had the highest proportion of lipids of differential association with FERMT2 between $\mathrm{AD}$ and control. It has been hypothesized that FERMT2 regulates APP internalization and degradation $^{53}$. For instance, FERMT2 silencing induces increased amounts of full-length APP and by-products and FERMT2 over-expression leads to a reduction of APP and its related metabolites. Recently, a direct interaction between FERMT2 and APP - through the F3 domain of FERMT2 and the NxTY motif within APP's intracellular domain was reported $^{54}$ and can influence axonal growth ${ }^{55}$. Importantly, because amyloidogenic processing of APP is dependent on lipid rafts, we postulate that the effect of FERMT2 might be-at least in part-due to alterations in lipid profiles which alter the accumulation of full-length APP or its by-products within the growth cone, impairing vesicle trafficking and/or cell adhesion.

$M S 4 A 6 A$ is membrane-spanning 4-domains, subfamily $\mathrm{A}$, member $6 \mathrm{~A}$ having been revealed to be associated with cortical and hippocampal atrophy independent of glucose metabolism and $\mathrm{A} \beta$ deposition ${ }^{56}$. The important association between $M S 4 A 6 A$ and PI (57.14\% had differential association with $M S 4 A 6 A)$ might indicate the involvement of PI mediating $M S 4 A 6 A$ 's effect on brain atrophy. Another gene $A B C A 7$ had an important effect on more than $50 \%$ of lipids in the DG lipid class, but with little influence on other lipid classes. DG metabolism and cholesterol synthesis might be the key component linking $A B C A 7$ to Alzheimer's onset. Increasing evidence has demonstrated that ABCA7 deficiency exacerbates $A \beta$ pathology in vitro and in vivo models. In detail, ABCA7 is involved in the microglial $A \beta$ clearance pathway and accelerated $\mathrm{A} \beta$ production ${ }^{57}$. ABCA7 may also mediate the release of cellular cholesterol and phospholipids to generate HDL. Human ABCA7 mediates the efflux of both cellular cholesterol and phospholipids to apoA-I, whereas mouse ABCA7 mediates only phospholipids but not cholesterol efflux to apoA-I ${ }^{58}$. Both $M S 4 A 6 A$ and $A B C A 7$ have been previously linked to the immune and complement system $^{59}$, lipid metabolism, and immune system $^{60}$, immune response and inflammation ${ }^{61}$, cytokine signaling in immune system ${ }^{62}$, cholesterol/lipid metabolism, and immune and complement systems ${ }^{63}$.

$C L U$-encoded apoliprotein $\mathrm{J}$ associated with the clearance of cellular debris and apoptosis, which was suggested to be involved in Abeta-independent pathways as part of the cascade leading to Alzheimer pathology ${ }^{64}$. In our study, SM and PE were revealed to be associated with CLU in the pathogenesis of $\mathrm{AD}$. ChE lipids were differentially associated with PICALM, SLC24A4, and SORL1 between $\mathrm{AD}$ and control. PICALM has been associated with reduced connectivity of the frontal gyrus with the hippocampus, as well as with the precuneus. The SLC24A4 gene encodes the 24 solute carrier family member 4 protein, which is a member of the $\mathrm{K}^{+}$-dependent $\mathrm{Na}^{+} / \mathrm{Ca}^{2+}$ exchanger protein family. These exchanger proteins are 
widely expressed in brain tissues, suggesting that SLC24A4 may play an important role in the nervous system. The association of this gene with blood pressure in African Americans suggests it may have relevance with $\mathrm{AD}$ through vascular disease ${ }^{65}$. SORL1 belongs to the lowdensity lipoprotein receptor family, binding ApoE and lipoprotein lipase on membranes ${ }^{66}$. Decreased Sorl1 has been identified as related to $\mathrm{AD}^{67}$. It is downregulated in lymphoblasts and cortical pyramidal neurons of $\mathrm{AD}$ patients. The mechanisms may include APP trafficking and recycling ${ }^{68}$, Additionally, the association of SORL1 with risk of amnestic mild cognitive impairment (aMCI) has been reported in the Han Chinese ${ }^{69}$. TG lipids were correlated with $B I N$, which functions in clathrin-mediated endocytosis and endocytic recycling, as does the AD risk gene PICALM. DNA methylation of the BIN1 promoter has been suggested as a possible epigenetic mechanism influencing AD risk.

Several prior studies have been conducted to explore the potential pathological roles of AD-related SNPs. A review of GWAS-identified risk genes for AD found they were clustered into three main molecular pathways: lipid metabolism, immune function, and endosome vesicle cycling ${ }^{70}$. The lipids that have shown marked differences between $\mathrm{AD}$ versus controls which been directly and indirectly linked to at least one of these mechanisms. The significant associations between lipid profiles and $\mathrm{AD}$ which were observed in our study encouraged us to further explore the possible mechanisms of lipids changes in $\mathrm{AD}$ patients.

Our results highlight that genetic pathway and SNPs related to AD influence lipids that are associated with $\mathrm{AD}$. These results reinforce the importance of lipid metabolism and dysregulation in $\mathrm{AD}$. It also suggests inflammation, ion channel modification, and $\mathrm{A} \beta$ pathways influence lipid levels that are dysregulated in AD. While studies examining the exact mechanisms linking these SNPs with reported lipid changes in $\mathrm{AD}$ patients are nascent in the current literature, our results provide renewed insights on potential lipid-related mechanisms in $\mathrm{AD}$ which may be examined further in preclinical and clinical studies.

\section{Limitations}

Firstly, the sample size was around 40 each in the AD and paired control group, which provides enough power for analyzing 9 lipid classes. However, the strength of the data is limited for examining a large number of individual lipids. FDR correction was used to reduce the bias, although a larger cohort is needed to replicate the findings.

Secondly, we did not subclassify AD to different stages. In addition, we did not look at overlapping cerebral vascular disease in $\mathrm{AD}$ patients because only 10 of $40 \mathrm{AD}$ subjects had MRI scanning. Therefore, future studies will look at lipid differences between control, pure $\mathrm{AD}$, and mixed AD in the same stage.
Thirdly, our study is useful for exploring clinical biomarkers of $\mathrm{AD}$ because plasma lipids are relatively easy to extract. However, plasma lipids may also be affected by damage to peripheral organs as well, which limits the accuracy of any association between changes in plasma lipid levels and brain pathology. The lipidomics of CSF or post-mortem brain samples from $\mathrm{AD}$ patients will be necessary to validate our findings in the future.

\section{Conclusion}

Our findings suggest that plasma lipids may differentiate between $\mathrm{AD}$ and cognitively normal controls and lipids models can be applied to discriminate the two groups. In addition, AD-related SNPs may have a different association with lipids between $\mathrm{AD}$ and controls and the underlying mechanisms need to be explored further in the future. In summary, our study provides evidence that certain lipids may be involved in $\mathrm{AD}$ pathogenesis and are associated with AD-related SNPs.

\section{Author details}

${ }^{1}$ Centre for Healthy Brain Ageing (CHeBA), School of Psychiatry, University of New South Wales, Sydney, Australia. ${ }^{2}$ Guangdong Mental Health Center, Guangdong Provincial People's Hospital, Guangdong Academy of Medical Sciences, Guangzhou, Guangdong, China. ${ }^{3}$ Neuroscience Research Australia, Randwick, Australia. ${ }^{4}$ Mark Wainwright Analytical Centre, University of New South Wales, Sydney, Australia. ${ }^{5}$ Neuropsychiatric Institute, Euroa Centre, Prince of Wales Hospital, Sydney, Australia. 'SChool of Medicine, Huzhou University, Huzhou Central Hospital Huzhou, Huzhou, China

\section{Conflict of interest}

The authors declare no competing interests.

\section{Publisher's note}

Springer Nature remains neutral with regard to jurisdictional claims in published maps and institutional affiliations.

Supplementary information The online version contains supplementary material available at https://doi.org/10.1038/s41398-021-01362-2.

Received: 26 June 2020 Revised: 10 March 2021 Accepted: 6 April 2021 Published online: 07 June 2021

References

1. Reitz, C., Brayne, C. \& Mayeux, R. Epidemiology of Alzheimer disease. Nat. Rev. Neurol. 7, 137-152 (2011).

2. Smith, M. A., Rottkamp, C. A., Nunomura, A., Raina, A. K. \& Perry, G. Oxidative stress in Alzheimer's disease. Biochim. Biophys. Acta 1502, 139-144 (2000).

3. Heppner, F. L., Ransohoff, R. M. \& Becher, B. Immune attack: the role of inflammation in Alzheimer disease. Nat. Rev. Neurosci. 16, 358-372 (2015).

4. $\mathrm{Xu}$, J. et al. Graded perturbations of metabolism in multiple regions of human brain in Alzheimer's disease: Snapshot of a pervasive metabolic disorder. Biochim. Biophys. Acta 1862, 1084-1092 (2016).

5. Morris, J. K., Honea, R. A., Vidoni, E. D., Swerdlow, R. H. \& Burns, J. M. Is Alzheimer's disease a systemic disease? Biochim. Biophys. Acta 1842, 1340-1349 (2014).

6. Dart, C. Lipid microdomains and the regulation of ion channel function. J. Physiol. 588, 3169-3178 (2010).

7. Brugger, B. Lipidomics: analysis of the lipid composition of cells and subcellular organelles by electrospray ionization mass spectrometry. Annu. Rev. Biochem. 83, 79-98 (2014). 
8. Walter, A. et al. Glycerophosphocholine is elevated in cerebrospinal fluid of Alzheimer patients. Neurobiol. Aging 25, 1299-1303 (2004).

9. Mapstone, M. et al. Plasma phospholipids identify antecedent memory impairment in older adults. Nat. Med. 20, 415-418 (2014)

10. Casanova, R. et al. Blood metabolite markers of preclinical Alzheimer's disease in two longitudinally followed cohorts of older individuals. Alzheimers Dement. 12, 815-822 (2016).

11. Sachdev, P. S. et al. The Sydney Memory and Ageing Study (MAS): methodology and baseline medical and neuropsychiatric characteristics of an elderly epidemiological non-demented cohort of Australians aged 70-90 years. Int. Psychogeriatr. 22, 1248-1264 (2010).

12. Wong, M. W. K. et al. Plasma lipidome variation during the second half of the human lifespan is associated with age and sex but minimally with BMI. PLoS ONE 14, e0214141 (2019).

13. Dahlgren, K. N. et al. Oligomeric and fibrillar species of amyloid-beta peptides differentially affect neuronal viability. J. Biol. Chem. 277, 32046-32053 (2002).

14. Meng, X. et al. Association between increased levels of amyloid-beta oligomers in plasma and episodic memory loss in Alzheimer's disease. Alzheimers Res Ther. 11, 89 (2019).

15. Wang, M. J. et al. Oligomeric forms of amyloid-beta protein in plasma as a potential blood-based biomarker for Alzheimer's disease. Alzheimers Res. Ther 9, 98 (2017)

16. Mather, K. A. et al. Investigating the genetics of hippocampal volume in older adults without dementia. PLOS ONE 10, e0116920 (2015)

17. Mather, K. A. et al. Genome-wide significant results identified for plasma apolipoprotein $\mathrm{H}$ levels in middle-aged and older adults. Sci. Rep. 6, 23675 (2016).

18. Euesden, J., Lewis, C. M. \& O'Reilly, P. F. PRSice: Polygenic Risk Score software Bioinformatics 31, 1466-1468 (2015).

19. Lambert, J. C. et al. Meta-analysis of 74,046 individuals identifies 11 new susceptibility loci for Alzheimer's disease. Nat. Genet. 45, 1452-1458 (2013).

20. Apostolova, L. G. et al. Associations of the Top 20 Alzheimer Disease Risk Variants With Brain Amyloidosis. JAMA Neurol. 75, 328-341 (2018).

21. Friedman, J., Hastie, T. \& Tibshirani, R. Regularization paths for generalized linear models via coordinate descent. J. Stat. Softw. 33, 1-22 (2010).

22. Team, R. C. R: A language and environment for statistical computing https:// www.R-project.org/ (2018)

23. Kuhn, M . et al. Caret: Classification and Regression Training. R package version. (2018)..

24. Robin, $\mathrm{X}$. et al. pROC: an open-source package for $\mathrm{R}$ and $\mathrm{S}+$ to analyze and compare ROC curves. BMC Bioinforma. 12, 77 (2011).

25. Podlisny, M. B. et al. Aggregation of secreted amyloid beta-protein into sodium dodecyl sulfate-stable oligomers in cell culture. J. Biol. Chem. 270, 9564-9570 (1995).

26. Seubert, P. et al. Isolation and quantification of soluble Alzheimer's betapeptide from biological fluids. Nature 359, 325-327 (1992).

27. Olsen, A. S. B. \& Faergeman, N. J. Sphingolipids: membrane microdomains in brain development, function and neurological diseases. Open Biol. 7, https:// doi.org/10.1098/rsob.170069 (2017)

28. Mielke, M. M. et al. Plasma ceramides are altered in mild cognitive impairment and predict cognitive decline and hippocampal volume loss. Alzheimers Dement. 6, 378-385 (2010)

29. Mielke, M. M. \& Lyketsos, C. G. Alterations of the sphingolipid pathway in Alzheimer's disease: new biomarkers and treatment targets? Neuromol. Med. 12, 331-340 (2010).

30. Varma, V. R. et al. Brain and blood metabolite signatures of pathology and progression in Alzheimer disease: a targeted metabolomics study. PLoS Med. 15, e1002482 (2018).

31. Han, X. et al. Metabolomics in early Alzheimer's disease: identification of altered plasma sphingolipidome using shotgun lipidomics. PLOS ONE 6, e21643 (2011)

32. Hussain, G. et al. Role of cholesterol and sphingolipids in brain development and neurological diseases. Lipids Health Dis. 18, 26 (2019).

33. Li, Z. et al. Reducing plasma membrane sphingomyelin increases insulin sensitivity. Mol. Cell. Biol. 31, 4205-4218 (2011).

34. Whiley, L. et al. Evidence of altered phosphatidylcholine metabolism in Alzheimer's disease. Neurobiol. Aging 35, 271-278 (2014).

35. Atri, A. Imaging of neurodegenerative cognitive and behavioral disorders: practical considerations for dementia clinical practice. Handb. Clin. Neurol. 136, 971-984 (2016).
36. Ojo, J. O. et al. Converging and differential brain phospholipid dysregulation in the pathogenesis of repetitive mild traumatic brain injury and Alzheimer's disease. Front. Neurosci. 13, 103 (2019).

37. Arancio, O. PIP2: a new key player in Alzheimer's disease. Cellscience 5, 44-47 (2008).

38. Sabogal-Guaqueta, A. M., Villamil-Ortiz, J. G., Arias-Londono, J. D. \& CardonaGomez, G. P. Inverse phosphatidylcholine/phosphatidylinositol levels as peripheral biomarkers and phosphatidylcholine/lysophosphatidylethanolaminephosphatidylserine as hippocampal indicator of postischemic cognitive impairment in rats. Front. Neurosci. 12, 989 (2018).

39. Gerl, M. J. et al. Cholesterol is inefficiently converted to cholesteryl esters in the blood of cardiovascular disease patients. Sci. Rep. 8, 14764 (2018).

40. Proitsi, P. et al. Plasma lipidomics analysis finds long chain cholesteryl esters to be associated with Alzheimer's disease. Transl. Psychiatry 5, e494 (2015).

41. Hejazi, L. et al. Mass and relative elution time profiling: two-dimensional analysis of sphingolipids in Alzheimer's disease brains. Biochem. J. 438, 165-175 (2011).

42. Wood, P. L. et al. Targeted lipidomics of fontal cortex and plasma diacylglycerols (DAG) in mild cognitive impairment and Alzheimer's disease: validation of DAG accumulation early in the pathophysiology of Alzheimer's disease. J. Alzheimer's Dis. 48, 537-546 (2015).

43. Shin, J., Xie, D. \& Zhong, X. P. MicroRNA-34a enhances T cell activation by targeting diacylglycerol kinase zeta. PLOS ONE 8, e77983 (2013).

44. Kalmijn, S. et al. Metabolic cardiovascular syndrome and risk of dementia in Japanese-American elderly men. The Honolulu-Asia aging study. Arterioscler. Thromb. Vasc. Biol. 20, 2255-2260 (2000).

45. Tajima, Y. et al. Lipidomic analysis of brain tissues and plasma in a mouse model expressing mutated human amyloid precursor protein/tau for Alzheimer's disease. Lipids Health Dis. 12, 68 (2013).

46. Ben Haim, L. \& Rowitch, D. H. Functional diversity of astrocytes in neural circuit regulation. Nat. Rev. Neurosci. 18, 31-41 (2017).

47. Jeong, W., Lee, H., Cho, S. \& Seo, J. ApoE4-induced cholesterol dysregulation and its brain cell type-specific implications in the pathogenesis of Alzheimer's disease. Mol. Cells 42, 739-746 (2019).

48. Zhang, J. \& Liu, Q. Cholesterol metabolism and homeostasis in the brain. Protein Cell 6, 254-264 (2015).

49. Oksanen, M. et al. Astrocyte alterations in neurodegenerative pathologies and their modeling in human induced pluripotent stem cell platforms. Cell. Mol. Life Sci. 76, 2739-2760 (2019).

50. Shulman, J. M. et al. Functional screening in Drosophila identifies Alzheimer's disease susceptibility genes and implicates Tau-mediated mechanisms. Hum. Mol. Genet. 23, 870-877 (2014).

51. Oksala, N. et al. Kindlin 3 (FERMT3) is associated with unstable atherosclerotic plaques, anti-inflammatory type II macrophages and upregulation of beta-2 integrins in all major arterial beds. Atherosclerosis 242, 145-154 (2015).

52. Ellison, J. A. et al. Osteopontin and its integrin receptor alpha(v)beta3 are upregulated during formation of the glial scar after focal stroke. Stroke 29, 1698-1706 (1998). discussion 1707.

53. Chapuis, J. et al. Genome-wide, high-content siRNA screening identifies the Alzheimer's genetic risk factor FERMT2 as a major modulator of APP metabolism. Acta Neuropathol. 133, 955-966 (2017).

54. Eysert, F. et al. Alzheimer's genetic risk factor FERMT2 (Kindlin-2) controls axonal growth and synaptic plasticity in an APP-dependent manner. Mol. Psychiatry https://doi.org/10.1038/s41380-020-00926-w (2020).

55. Sosa, L. J. et al. Amyloid precursor protein is an autonomous growth cone adhesion molecule engaged in contact guidance. PLOS ONE 8, e64521 (2013).

56. Ramirez, L. M. et al. Common variants in ABCA7 and MS4A6A are associated with cortical and hippocampal atrophy. Neurobiol. Aging 39, 82-89 (2016).

57. Aikawa, T., Holm, M. L. \& Kanekiyo, T. ABCA7 and pathogenic pathways of Alzheimer's disease. Brain Sci. 8, https://doi.org/10.3390/brainsci8020027 (2018).

58. Wang, X. \& Paigen, B. Genetics of variation in HDL cholesterol in humans and mice. Circ. Res. 96, 27-42 (2005).

59. Lambert, J. C. et al. Genome-wide association study identifies variants at CLU and CR1 associated with Alzheimer's disease. Nat. Genet. 41, 1094-1099 (2009)

60. Sims, R. et al. Rare coding variants in PLCG2, ABI3, and TREM2 implicate microglial-mediated innate immunity in Alzheimer's disease. Nat. Genet. 49, 1373-1384 (2017) 
61. MahmoudianDehkordi, S. et al. Altered bile acid profile associates with cognitive impairment in Alzheimer's disease-An emerging role for gut microbiome. Alzheimers Dement. 15, 76-92 (2019).

62. Walker, D. G., Tang, T. M. \& Lue, L. F. Studies on colony stimulating factor receptor-1 and ligands colony stimulating factor-1 and interleukin-34 in Alzheimer's disease brains and human microglia. Front. Aging Neurosci. 9, 244 (2017).

63. Hollingworth, P. et al. Common variants at ABCA7, MS4A6A/MS4A4E, EPHA1, CD33 and CD2AP are associated with Alzheimer's disease. Nat. Genet. 43, 429-435 (2011).

64. Slot, R. E. R. et al. ApoE and clusterin CSF levels influence associations between APOE genotype and changes in CSF tau, but not CSF Abeta42, levels in nondemented elderly. Neurobiol. Aging 79, 101-109 (2019).
65. Adeyemo, A. et al. A genome-wide association study of hypertension and blood pressure in African Americans. PLoS Genet. 5, e1000564 (2009).

66. Jacobsen, L. et al. Activation and functional characterization of the mosaic receptor SorLA/LR11. J. Biol. Chem. 276, 22788-22796 (2001).

67. Scherzer, C. R. et al. Loss of apolipoprotein E receptor LR11 in Alzheimer disease. Arch. Neurol. 61, 1200-1205 (2004).

68. Rogaeva, E. et al. The neuronal sortilin-related receptor SORL1 is genetically associated with Alzheimer disease. Nat. Genet. 39, 168-177 (2007)

69. Gao, X. et al. SORL1 genetic variants modulate risk of amnestic mild cognitive impairment in northern Han Chinese. Int. J. Neurosci. 124, 296-301 (2014).

70. Van Cauwenberghe, C., Van Broeckhoven, C. \& Sleegers, K. The genetic landscape of Alzheimer disease: clinical implications and perspectives. Genet. Med. 18, 421-430 (2016). 